\title{
On Addressing the Security and Stability Issues Due to False Data Injection Attacks in DC Microgrids An Adaptive Observer Approach
}

Cecilia, Andreu; Sahoo, Subham; Dragicevic, Tomislav; Costa-Castello, Ramon; Blaabjerg, Frede

Published in:

IEEE Transactions on Power Electronics

Link to article, DOI:

10.1109/TPEL.2021.3114990

Publication date:

2021

Document Version

Peer reviewed version

Link back to DTU Orbit

Citation (APA):

Cecilia, A., Sahoo, S., Dragicevic, T., Costa-Castello, R., \& Blaabjerg, F. (2021). On Addressing the Security and Stability Issues Due to False Data Injection Attacks in DC Microgrids An Adaptive Observer Approach. IEEE Transactions on Power Electronics, 37(3), 2801 - 2814. https://doi.org/10.1109/TPEL.2021.3114990

\section{General rights}

Copyright and moral rights for the publications made accessible in the public portal are retained by the authors and/or other copyright owners and it is a condition of accessing publications that users recognise and abide by the legal requirements associated with these rights.

- Users may download and print one copy of any publication from the public portal for the purpose of private study or research.

- You may not further distribute the material or use it for any profit-making activity or commercial gain

- You may freely distribute the URL identifying the publication in the public portal 


\title{
On Addressing the Security and Stability Issues Due to False Data Injection Attacks in DC Microgrids - An Adaptive Observer Approach
}

\author{
Andreu Cecilia, Student Member, IEEE, Subham Sahoo, Member, IEEE, Tomislav Dragičević, Senior Member, \\ IEEE, Ramon Costa-Castelló, Senior Member, IEEE and Frede Blaabjerg, Fellow, IEEE
}

\begin{abstract}
This paper proposes an observer-based methodology to detect and mitigate false data injection attacks in collaborative DC microgrids. The ability of observers to effectively detect such attacks is complicated by the presence of unknown nonlinear constant power loads. This work determines that, in the presence of unknown constant power loads, the considered attack detection and mitigation problem involves non-linearities, locally unobservable states, unknown parameters, uncertainty and noise. Taking into account these limitations, a distributed non-linear adaptive observer is proposed to overcome these limitations and solve the concerned observation problem. The necessary conditions for the stability of the distributed scheme are found out. Moreover, numerical simulations are performed and then validated in a real experimental prototype, where communication delay, uncertainty and noise are considered.
\end{abstract}

Index Terms-Cyber-attacks, DC microgrid, constant power loads, non-linear observer, cyber-physical systems, resilient controller, adaptive observer, distributed estimation.

\section{INTRODUCTION}

$\mathbf{T}$ he DC nature of renewable energy sources (e.g. fuel cells [1] or photovoltaic panels [2]) has motivated the further development of DC microgrids in order to avoid redundant conversions along the power grid. Adequate DC microgrid operation requires equal current sharing between the distributed generation units (DGUs), as well as stable and accurate DC voltage control [3]-[4]. One of the commonly used coordination framework between DGUs is the distributed framework, which relaxes the scalability issues, offers high bandwidth and resilience against the single point of failure [5].

Consequently, the microgrid control relies on the integration of a communication cyber-layer to improve its performance [6]. The interaction between the physical-layer and the cyber-layer creates a risk of malicious cyber-attacks, which may endanger the performance of the DGUs and/or the whole DC microgrid.

This work has been partially funded by the Spanish State Research Agency through the María de Maeztu Seal of Excellence to IRI (MDM-2016-0656) and by the VELUX FOUNDATIONS under the VILLUM Investigator grantREPEPS (Award 00016591).

A. Cecilia and R. Costa-Castelló are with the Institut de Robòtica i Informàtica Industrial, CSIC-UPC Llorens i Artigas 4-6, 08028 Barcelona, Spain (e-mail: andreu.cecilia@upc.edu and ramon.costa@upc.edu). (Corresponding author: Andreu Cecilia)

S. Sahoo and F. Blaabjerg are with the Department of Energy Technology, Aalborg University, Aalborg East, 9220, Denmark (e-mail: sssa@et.aau.dk and fbl@et.aau.dk).

T. Dragičević is with the Center of Electric Power and Energy,Technical University of Denmark, 2800 Kgs. Lyngby, Denmark (email:tomdr@elektro.dtu.dk).
For this reason, the interest in developing attack detection and mitigation techniques, from a control viewpoint, has increased in the recent years [7].

Amongst many different types of cyber-attacks, e.g. false data injection attacks (FDIAs) [8], denial of service [9] and replay attacks [10]; this work focuses on the FDIA as it is the most prominent cyber-attack [8]. This attack is conducted by injecting malicious data in hijacked measurements in order to modify the behaviour of the microgrid.

Reliable security measures against FDIAs rely on detecting if an attack is present or not in the system and identifying the compromised agent. In this context, a successful strategy is based on implementing an observer that is independent of the attack signal and a detector that computes the presence of the attack by comparing the estimation with the measured signals. Some examples of this approach may be the use of a weighted least squares as the observer and a sparse optimization as a detector [11], the use of Kullback-Leibler distance as the detector, a Kalman filter as the observer and a Euclidean distance as the detector [12], a Kalman filter with a cosine similarity approach as detector [13], [14], neural-network based detector [15] or a short-term state forecasting as the observer [16]. One of the main drawbacks of the mentioned approaches is that the observer and the detector were implemented in a centralized framework, which hinders the scalability of the solution to large systems. Consequently, recent detection algorithms are changing to the distributed framework [17]. Some notable examples are the use of constant gain distributed linear observers [18], distributed extended Kalman filters [19], [20], a bank of unknown input observers [21] and distributed sliding mode observers [22]. For a more in-depth review of the potential impacts, vulnerabilities, and detection strategies of FDIAs in power systems, the reader is referred to the surveys in [23], [24] and references within.

Once a FDIA has been detected, the immediate objective is to mitigate the influence of the attack on the microgrid without a significant effect on the system performance [25]. In relation to DC microgrids, an attack estimation approach has been proposed in [22], an event-driven approach has been proposed to mitigate FDIA [26], man-in-the-middle attacks for a system of homogeneous agents [27] and for a system of heterogeneous agents [28].

A major limitation of available observer-based detection and mitigation methods is the assumption that the microgrid's dynamics are linear. In many cases, the load side converter 
is required to deliver a constant power to the load. In such situations, the voltage dynamics behave non-linearly [29], where small deviations of the load voltage may produce large variations of the equilibrium point, thereby limiting the linear approximations. Moreover, it is reasonable to assume that the constant power load (CPL) is not known, which makes the dynamics linearization process infeasible.

Furthermore, it is well-known that CPLs induce non-linear dynamics and have a destabilizing effect on the DC microgrid. In such context, the interaction of FDIAs and CPLs may drive the power system to unstable equilibrium points, which may lead to significant oscillations or to network collapse. This instability behaviour cannot be replicated in linear DC microgrids with the same false data signal. An example of this fact is presented in the subsection "II-A Impact on stability due to FDIA in presence of CPLs".

The purpose of the present work is to design a distributed non-linear observer that can be used to reconstruct the false data in DC microgrids comprising of unknown CPLs. The reconstruction of an attack signal is a more prohibitive process than the detection and identification of the cyber-attack, which only requires finding the compromised agent and does not necessarily compute the actual FDIA signal. However, it offers a set of advantages. In particular, the estimated attack signal value can be used to remove the false data from the compromised measurement(s), which can serve the dual purpose of addressing both security of the system, by detecting the presence of false data, and stability conflicts in the microgrid, by extracting the false data from the system before stability issues arise.

A solution for a similar problem has been recently proposed in [22]. Despite presenting promising results, the approach had some conflicts that limited its practical implementation. First, the states are estimated through a sliding-mode observer, which is known to be highly sensitive to measurement noise [30]. Second, the observer relied on high-gain feedback terms in order to cancel the effect of non-local states, this fact also increments the noise sensitivity of the observer and hinders its transient performance. Third, the estimation of the CPL is based on appending the CPL in the state vector as a constant variable and implementing an observer for the extended state. This approach is highly sensitive to model uncertainty and measurement noise. Finally, the power line currents were estimated through an open-loop integration, consequently, the accuracy of the FDIA estimation was highly sensitive to uncertainty in the power line parameters and the convergence rate of the estimation was not tunable. As the proposed observer was highly sensitive to measurement noise, it required the implementation of low-pass filters, which incremented the phase-lag of the estimator. This fact limited its capability of estimating time-varying FDIAs. Consequently, this work proposes a new observer structure that addresses all the commented issues and, as a consequence, presents significantly higher performance than the solution proposed in [22].

Specifically, the main contributions of this work are:

- A distributed non-linear adaptive observer-based strategy that achieves a reliable state-estimation for DC microgrid models comprising of unknown CPLs.

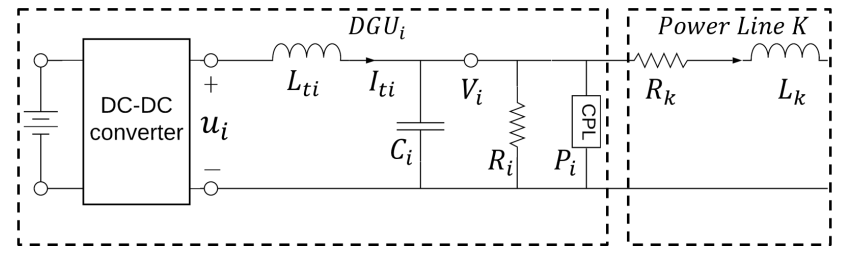

Fig. 1. Electrical scheme of the DGU and power line k. Used symbols are described in Table I.

TABLE I

SYMBOLS USED IN FIG. 1

\begin{tabular}{|c|c|}
\hline States & \\
\hline$I_{t i}$ & DGU output current \\
$V_{i}$ & Voltage \\
$I_{k}$ & Power line current \\
\hline Parameters & \\
\hline$L_{t i}$ & Filter inductance \\
$C_{i}$ & Shunt capacitor \\
$R_{i}$ & Local load impedance \\
$R_{k}$ & Power line resistance \\
$L_{k}$ & Power line inductance \\
\hline Inputs & \\
\hline$u_{i}$ & Converter voltage \\
$P_{i}$ & CPL \\
\hline
\end{tabular}

- The necessary conditions for the stability of the estimation scheme are given.

- The secure estimation is used to detect and reconstruct the false data.

- The viability of the mitigation scheme is studied through a numerical simulation and in a experimental prototype, where sensor noise, uncertainty and communication delay are considered.

The remainder of this paper is organized as follows. Section II introduces the concerned cooperative DC microgrid model and formulates the attack mitigation problem. Section III presents the non-linear adaptive observer algorithm that is used for the attack reconstruction. In Section IV, the proposed approach is validated in a set of numerical simulations. In Section V, the approach is validated in a real experimental prototype. Finally, Section VI draws some conclusions.

\section{SYSTEM'S MODEL AND PROBLEM FORMULATION}

This work considers a DC microgrid composed by a set of DGUs. The different DGUs are connected through a set of resistive power lines. Each DGU is comprised of a DC voltage source and a DC-DC converter. Moreover, the DGU supplies power to a CPL and a constant impedance. The electrical scheme of the proposed DGU model is depicted in Fig. 1.

This work considers an averaged model of the DC-DC converter, which results in the following equations for the dynamics of the $i_{t h}$ DGU:

$$
\begin{aligned}
L_{t i} \dot{I}_{t i} & =-V_{i}+u_{i} \\
C_{i} \dot{V}_{i} & =I_{t i}-\sum_{k \in \mathscr{E}_{i}} I_{k, i}-\frac{1}{R_{i}} V_{i}-P_{i} \frac{1}{V_{i}} \\
L_{k} \dot{I}_{k} & =\left(V_{i}-V_{j}\right)-R_{k} I_{k} \quad \forall k \in \mathscr{E}_{i},
\end{aligned}
$$


where $u_{i}$ depicts the output voltage of the converter and $\mathscr{E}_{i}$ is the set of incident power lines.

It is assumed that there are sensors that can measure the generated current, $I_{t i}$, and the load voltage, $V_{i}$, but, no sensor is deployed to estimate the line current, $I_{k}$, since this is not a control quantity used for control of DGUs. Therefore, the output in the $i_{t h}$ DGU is defined as $\mathbf{y}_{i}=\left[y_{1, i}, y_{2, i}\right]^{\top}=\left[I_{t i}, V_{i}\right]^{\top}$.

In the cyber-layer, the DC microgrid is modeled through an undirected and connected communication graph $\mathscr{G}=\{\mathscr{V}, \mathscr{E}\}$, where $\mathscr{V}$ depicts the set of DGU and $\mathscr{E}$ depicts the power lines between the DGUs [31]. The graph is described through an incident matrix $\mathscr{B} \in \mathbb{R}^{n \times m}$, where $n$ is the number of DGUs and $m$ the number of resistive power lines, defined as:

$$
\mathscr{B}_{i j}= \begin{cases}+1, & \text { if } \mathrm{i} \text { is the positive end of the line } \mathrm{j} \\ -1, & \text { if } \mathrm{j} \text { is the negative end of the line } \mathrm{j} \\ 0, & \text { otherwise. }\end{cases}
$$

where $\mathscr{B}_{i j}$ are the components of the matrix $\mathscr{B}$. For the adequate operation of the DC microgrid, each DGU is only required to send and receive information from its neighbour DGUs. Consequently, the communication graph in the cyberlayer coincides with the physical power network of the microgrid. Nonetheless, the efficacy of the proposed method is not based on the fact that the power network graph is the same as the communication graph.

As an assumption, the DC microgrid is controlled through the droop control philosophy in order to ensure equal current sharing among the agents. It operates with an error across the voltage reference. To compensate for this error, secondary controllers are employed to provide compensation terms to limit the offset [32]. This work considers the most common case, that is each DGU has two PI controllers connected in cascade that ensure the tracking of the voltage reference and current control. Therefore, the input voltage, $u_{i}$, is generated through the following PI controller [33]

$$
u_{i}=K_{p I}\left(y_{1, i}-I_{\text {ref }, i}\right)+K_{i I} \int\left(y_{1, i}-I_{\text {ref }, i}\right) .
$$

where $K_{p I}$ is the gain of the proportional part, $K_{i I}$ is the gain of the integral part and $I_{r e f, i}$ is the current reference generated by the previous PI in the cascaded controller.

The PI controller ensures that the DGU's load voltage and the DGU output current present bounded trajectories. Moreover, the PI controller includes saturation limits that prevents under/over-voltage. A more in-depth description of the controller is presented in [22].

In the distributed control topology, each DGU local control is complemented by the information from cyber-layer neighbouring DGUs to establish a distributed coordination. Between DGUs, the information vector $\boldsymbol{\psi}_{i}=\left[\psi_{1, i}, \psi_{2, i}\right]^{\boldsymbol{\top}}=\left[\hat{v}_{d c, i}, I_{t i}\right]^{\top}$ is transmitted, where $\hat{v}_{d c, i}$ depicts the average voltage estimate in the $i_{t h}$ DGU [4]. The information vector, $\boldsymbol{\psi}_{i}$, is used to create a voltage off-set to be compensated by the secondary controllers [5]. More precisely, the local voltage reference, $V_{d c, r e f}$, to be tracked by the $i_{t h}$ DGU is disturbed as follows:

$$
V_{d c, r e f, i}=V_{d c, r e f}+\Delta V_{1 i}+\Delta V_{2 i}
$$

where $\Delta V_{1 i}$ and $\Delta V_{2 i}$ are the two voltage off-set computed as:

$$
\begin{aligned}
& \Delta V_{1 i}=H_{1}(s)\left(V_{d c, r e f}-\sum_{k \in \mathscr{E}_{i}}\left(\hat{v}_{d c, k}-\hat{v}_{d c, i}\right)\right) \\
& \Delta V_{2 i}=H_{2}(s)\left(I_{d c, r e f}-\sum_{k \in \mathscr{E}_{i}}\left(I_{t k}-I_{t i}\right)\right)
\end{aligned}
$$

where $I_{d c, r e f}$ is a global current reference quantities for the whole microgrid, and $H_{1}(s)$ and $H_{2}(s)$ are proportional integral controllers.

A depiction of the presented control can be seen in the left hand side of Fig. 2.

This paper focuses on the reconstruction of false data that can affect the DGU output current measurements. Explicitly, an attack on the $i_{t h}$ DGU is depicted as:

$$
\begin{aligned}
\text { Sensor attack : } & \mathbf{y}_{i}=\left[I_{t i}+x_{i}^{a}, V_{i}\right]^{\top} \\
\text { Cyber-link attack : } & \boldsymbol{\psi}_{i}=\left[\hat{v}_{d c, i}, I_{t i}+x_{i}^{a}\right]^{\top}
\end{aligned}
$$

where $x_{i}^{a}$ represents the FDIA value.

It is assumed that the FDIA can be classified as a deception attack [33], which means that it satisfies the instantaneous system objectives but may affect the performance of the microgrid later.

It is also assumed that the voltage sensor is free of attacks, as previous works have proved that a stealth attack is not possible by manipulating the voltage due to the presence of a distributed observer [22], [34].

The main objective is to design an observer that can estimate the attack signal, $x_{i}^{a}$. After that, the reconstructed attack signal can be used to mitigate the FDIA. Precisely, if an attack in the sensor is exactly estimated, i.e. $x_{i}^{a}=\hat{x}_{i}^{a}$, later, the FDIA can be removed from the microgrid as follows:

$$
\mathbf{y}_{i}^{\text {cleaned }}=\left[y_{1, i}-\hat{x}_{i}^{a}, y_{2, i}\right]^{\boldsymbol{\top}}=\left[I_{t i}, V_{i}\right]^{\top} .
$$

A scheme of the proposed reconstruction and mitigation strategy is depicted in Fig. 2.

This mitigation algorithm is planned to be implemented in large scale systems. For reasons stated before, it is of high interest to implement the observer in a distributed framework. Consequently, the observer implemented in the $i_{t h}$ DGU has to estimate the DGU output current, $I_{t i}$, with only the signals measured in the $i_{t h}$ DGU, $\mathbf{y}_{i}$, and the signals transmitted through the neighbour DGUs.

In order to reconstruct the current, it is required to design a non-linear observer through the direct study of the non-linear model in (1). Consider the following:

Lemma II.1. The $i_{t h} D G U$ output current, $I_{t i}$, can be computed through the input $u_{i}$, the voltage $V_{i}$, its derivative, $\dot{V}_{i}$, the $C P L, P_{i}$, and the line currents, $I_{k, i}$, by computing the following expression

$$
\hat{I}_{t i}=C_{i} \dot{\hat{V}}_{i}+\sum_{k \in \mathscr{E}_{i}} \hat{I}_{k, i}+\frac{1}{R_{i}} V_{i}+\hat{P}_{i} \frac{1}{V_{i}} .
$$

Proof. Expression (6) is obtained by isolating $I_{t i}$ from the second equation in (1). 


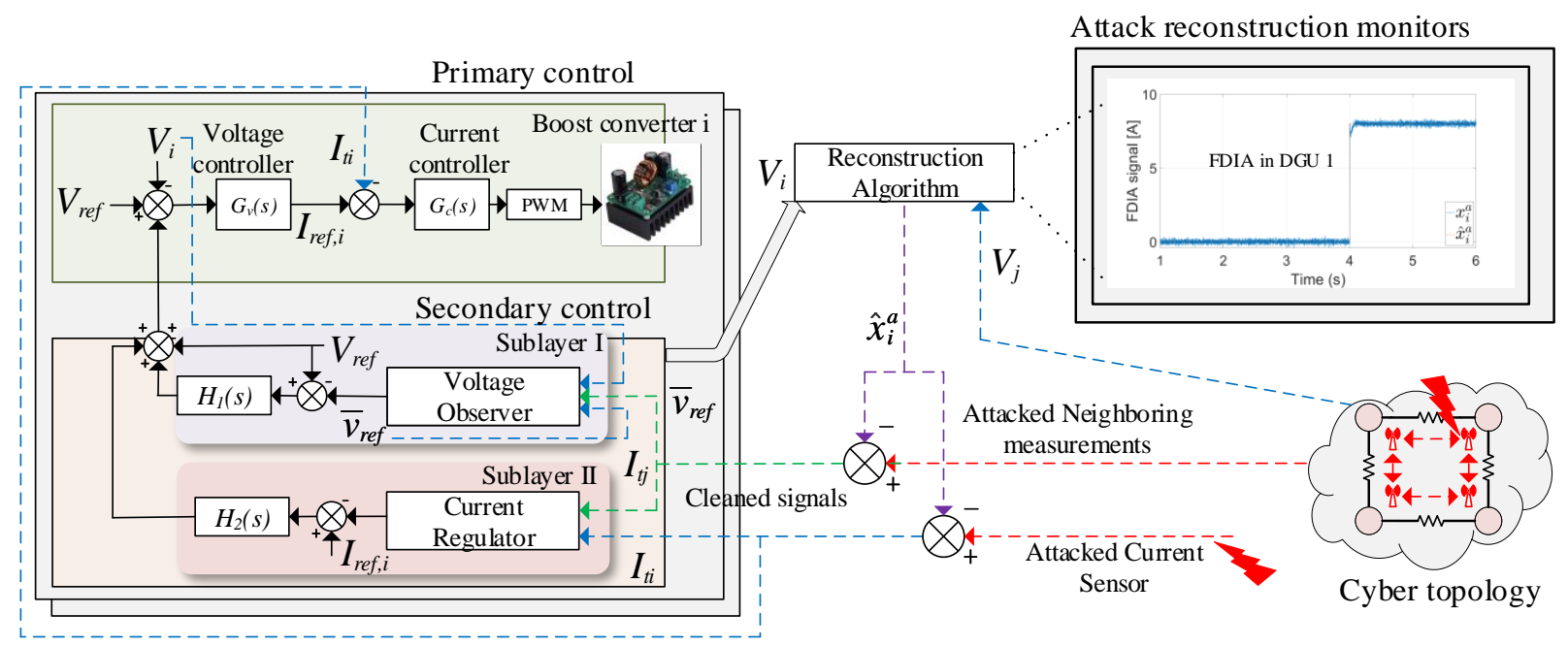

Fig. 2. General scheme of the proposed FDIA mitigation strategy. More details of primary and secondary controller can be found in [22].

Therefore, assuming that one generates an estimation, $\dot{\hat{V}}_{i}$ $\hat{I}_{k, i}$ and $\hat{P}_{i}$, then, $\left\|\hat{I}_{t i}-I_{t i}\right\| \rightarrow 0$, where $\hat{I}_{t i}$ is computed through (6) using the estimations $\dot{\hat{V}}_{i}, \hat{I}_{k, i}$ and $\hat{P}_{i}$.

\section{A. Impact on stability due to FDIA in presence of CPLs}

The concerned model presents strongly nonlinear dynamics induced by the CPLs that may contribute to the microgrid instability by the non-linearity of the CPL. As stated before, the DGUs are commonly controlled through linear PI controllers, as a consequence, the stability of the agent can only be ensured inside region of attraction around the operating point considered during the PI tuning [35]. Precisely, there is a region $D_{i} \subset \mathbb{R}^{2}$ such that if $I_{t i}, V_{i} \in D_{i}$, then, the DGU'states converge to the desired trajectories. Otherwise, the agent becomes unstable or presents large oscillations [35]. For the concerned microgrid, the region of attraction can be approximated a series of sum of square optimizations [35].

The injection of a FDIA induces a large variation of the state variables that may lead to an escape of the region of attraction and, consequently, destabilize the system. This fact confirms that the interaction between FDIAs and CPLs can destabilize the plant.

An experimental example of this statement is presented in Fig. 3, where a DC microgrid of 2 DGUs with CPLs $(782 \mathrm{~W})$ is tampered by a small step function FDIA. When an attack element of $x_{a}^{1}=0.8 \mathrm{~A}$ is introduced in the agent 1 , the average voltage remains unaltered, while an oscillatory instability is induced in the DGU current. Such phenomenon does not occur in the system with linear dynamics, i.e. in the absence of CPLs. Indeed, if the same FDIA is conducted in a similar microgrid without CPLs, the performance of the system is not significantly affected.

\section{Proposed Non-Linear Observer}

The objective here is to design an observer algorithm that can accurately estimate, $\dot{V}_{i}, I_{k, i}$ for $k \in \mathscr{E}_{i}$ and $P_{i}$, of the $i_{t h}$ DGU,

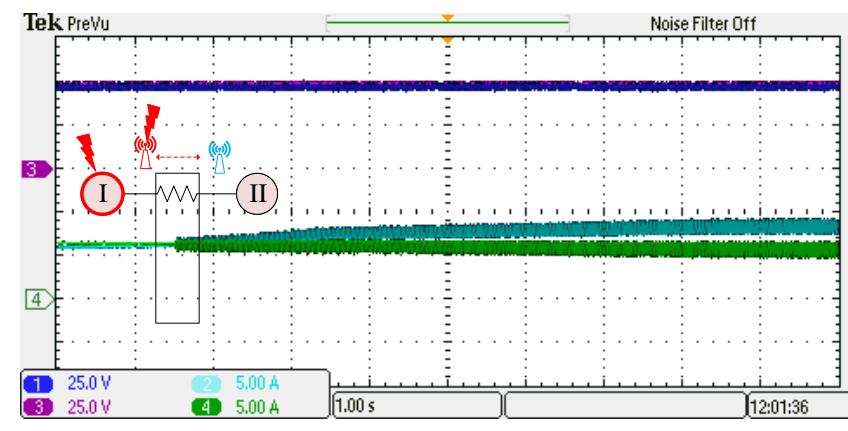

Fig. 3. Experimental example of oscillatory behaviour induced by a step FDIA in presence of CPLs. At time $t=1.5 \mathrm{~s}$, false data $x_{a}^{1}=0.8 \mathrm{~A}$ is introduced in the measurements of Agent 1.

even in the presence of false data, unmodelled disturbances, model uncertainty and sensor noise.

First, it is convenient to define a coordinate change that transforms the system into a form that eases the observability study and observer design.

Lemma III.1. The following input-independent map:

$$
\left[\begin{array}{c}
\xi_{1, i} \\
\xi_{2, i} \\
\eta_{1, i} \\
\vdots \\
\eta_{m, i}
\end{array}\right]=\varphi\left(V_{i}, \dot{V}_{i}, I_{1, i}, \ldots, I_{m_{i}, i}\right)=\left[\begin{array}{c}
V_{i} \\
\dot{V}_{i} \\
I_{1, i} \\
\vdots \\
I_{m_{i}, i}
\end{array}\right]
$$

defines a diffeomorphism that transforms the system (1) into the following triangular form

$$
\begin{aligned}
& \dot{\xi}_{1, i}=\xi_{2, i} \\
& \dot{\xi}_{2, i}=\phi_{i}\left(\boldsymbol{\xi}_{i}, u_{i}, P_{i}, \boldsymbol{\eta}_{i}\right)+w_{1} \\
& \dot{\eta}_{j, i}=\frac{1}{L_{k}}\left(\xi_{1, i}-\xi_{1, j}\right)-\frac{R_{k}}{L_{k}} \eta_{j, i}+w_{2, j} \quad \text { for } j=1, \ldots, m_{i}
\end{aligned}
$$




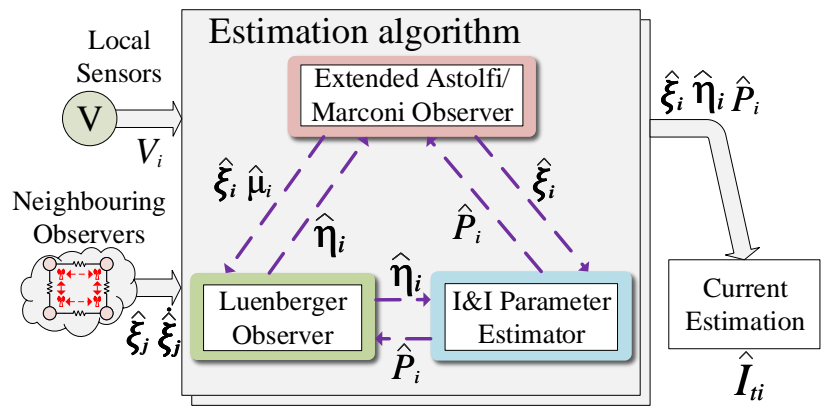

Fig. 4. Scheme of the proposed non-linear adaptive observer.

where $\boldsymbol{\xi}_{i}=\left[\xi_{1, i}, \xi_{2, i}\right]^{\top}$ are the local voltage and its derivative, $\boldsymbol{\eta}_{i}=\left[I_{1, i}, \ldots I_{m_{i}, i}\right]^{\top}$ are the incident power line currents, $w_{1}$ and $w_{2, j}$ for $j=1, \ldots, m_{i}$ represent unknown disturbances or model uncertainty and

$$
\begin{gathered}
\phi_{i}\left(\boldsymbol{\xi}_{i}, u_{i}, P_{i}, \boldsymbol{\eta}_{i}\right)=\frac{1}{C_{i}}\left(\frac{1}{L_{t i}}\left(-\xi_{1, i}+u_{i}\right)+P_{i} \frac{\xi_{2, i}}{\xi_{1, i}^{2}}\right. \\
\left.-\sum_{k \in \mathscr{E}_{i}}\left(\frac{1}{L_{k}}\left(\xi_{1, i}-\xi_{1, j}\right)-\frac{R_{k}}{L_{k}} \eta_{k, i}\right)-\frac{1}{R_{i}} \xi_{2, i}\right) .
\end{gathered}
$$

The measured signal in the new coordinates is $\boldsymbol{y}=V_{i}=\xi_{1, i}$.

Proof. The Jacobian of $\varphi\left(V_{i}, \dot{V}_{i}, I_{1, i}, \ldots, I_{m_{i}, i}\right)$ is full rank in all DGU operating conditions, therefore, the function $\phi_{i}$ defines a diffeomorphism in the considered operating conditions.

By inspection of the structure in (8), it is possible to study the elements involved in the estimation problem that prevents the direct implementation of solutions available in the literature.

First, for system in (8), if the local voltage, $V_{i}=\xi_{1, i}$, is the measured signal, most observer techniques, e.g. the slidingmode observer in [22], can only achieve an estimation of $\xi_{1, i}$ and $\xi_{2, i}$, as all the power line currents as $\boldsymbol{\eta}_{i}$ are in the unobservable space of the system [36]. Second, standard robust observers give no information about the unknown CPL, $P_{i}$, and may present noise sensitivity problems. Finally, the inability of estimating the CPL, $P_{i}$, and the power line currents, $I_{k}$, prevents the computation of (6), which makes the proposed mitigation strategy difficult to implement.

To solve these limitations, this work proposes designing the observer as the interconnection of three sub-systems. First, an extended Astolfi/Marconi observer [37], [38] will be designed to robustly estimate the states $\xi_{1, i}, \xi_{2, i}$ and a virtual state, $\sigma$. Second, the virtual state, $\sigma$, will be used to compute an auxiliary signal, $\mu_{i}$, that is going to be implemented as the measured signal in a Luenberger observer to robustly estimate the unobservable power line currents, $\boldsymbol{\eta}_{i}$. Finally, the unknown power load will be estimated through an adaptive law based on the immersion and invariance (I\&I) technique [39]. The reasoning behind such observer structure will be explained throughout this paper. A general scheme of the proposed observer algorithm is depicted in Fig. 4.
A. Estimation of $\xi_{1, i}, \xi_{2, i}$ and design of extended Astolf/Marconi observer

Let us consider the first two equations of (8), and extend the system as follows:

$$
\begin{aligned}
\dot{\xi}_{1, i} & =\xi_{2, i} \\
\dot{\xi}_{2, i} & =\sigma \\
\dot{\sigma} & =\dot{\phi}_{i}\left(\boldsymbol{\xi}_{i}, \dot{u}_{i}, P_{i}, \boldsymbol{\eta}_{i}, \boldsymbol{\xi}_{2, k}\right)+\dot{w}_{1}
\end{aligned}
$$

where

$$
\begin{aligned}
& \dot{\phi}_{i}\left(\boldsymbol{\xi}_{i}, \dot{u}_{i}, P_{i}, \boldsymbol{\eta}_{i}, \boldsymbol{\xi}_{2, k}\right)= \\
& \frac{\partial \phi_{i}\left(\boldsymbol{\xi}_{i}, u_{i}, P_{i}, \boldsymbol{\eta}_{i}\right)}{\partial \boldsymbol{\xi}_{i}} \dot{\boldsymbol{\xi}}_{i}+\frac{\partial \phi_{i}\left(\boldsymbol{\xi}_{i}, u_{i}, P_{i}, \boldsymbol{\eta}_{i}\right)}{\partial \boldsymbol{\eta}_{i}} \dot{\boldsymbol{\eta}}_{i} \\
& +\frac{\partial \phi_{i}\left(\boldsymbol{\xi}_{i}, u_{i}, P_{i}, \boldsymbol{\eta}_{i}\right)}{\partial u_{i}} \dot{u}_{i}+\sum_{k \in \mathscr{E}_{i}} \frac{\partial \phi_{i}\left(\boldsymbol{\xi}_{i}, u_{i}, P_{i}, \boldsymbol{\eta}_{i}\right)}{\partial \xi_{1, k}} \xi_{2, k} .
\end{aligned}
$$

The objective is to design an algorithm to estimate $\xi_{1, i}, \xi_{2, i}$ and $\sigma$ in (10). System in (10) is uniformly observable in the inputs, $u_{i}$, [40], which allows the implementation of nonlinear observers as the high-gain observer [41] or sliding-mode observer [22]. However, the convergence rate and robustness of said observers relies on increasing the gain in the feedback term, which significantly increases the observer's sensor noise sensitivity [41], i.e. small noise in $y$ can significantly aggravate the state-estimation accuracy.

For this reason, this work proposes implementing an Astolfi/Marconi low-power observer [37]. The Astolfi/Marconi observer includes some extra dynamics that attenuates the noise effect on the estimation accuracy without losing convergence rate and robustness properties [37].

For the concerned triangular structure (10), such observer takes the following form

$$
\begin{aligned}
& \dot{\hat{\xi}}_{1, i}=\lambda_{1, i}+\frac{\alpha_{1}}{\varepsilon}\left(y-\hat{\xi}_{1, i}\right) \\
& \dot{\hat{\xi}}_{2, i}=\lambda_{2, i}+\frac{\alpha_{2}}{\varepsilon}\left(\lambda_{1, i}-\hat{\xi}_{2, i}\right) \\
& \dot{\hat{\sigma}}=\dot{\phi}_{i}\left(\hat{\boldsymbol{\xi}}_{i}, \dot{u}_{i}, \hat{P}_{i}, \hat{\boldsymbol{\eta}}_{i}, \hat{\boldsymbol{\xi}}_{2, i}\right)+\frac{\alpha_{3}}{\varepsilon}\left(\lambda_{2, i}-\hat{\sigma}\right) \\
& \dot{\lambda}_{1, i}=\lambda_{2, i}+\frac{\beta_{1}}{\varepsilon^{2}}\left(y-\hat{\xi}_{1, i}\right) \\
& \dot{\lambda}_{2, i}=\dot{\phi}_{i}\left(\hat{\boldsymbol{\xi}}_{i}, \dot{u}_{i}, \hat{P}_{i}, \hat{\boldsymbol{\eta}}_{i}, \hat{\boldsymbol{\xi}}_{2, k}\right)+\frac{\beta_{2}}{\varepsilon^{2}}\left(\lambda_{1, i}-\hat{\xi}_{2, i}\right)
\end{aligned}
$$

where $\hat{\boldsymbol{\xi}}_{i}=\left[\hat{\xi}_{1, i}, \hat{\xi}_{2, i}\right]^{\top}$ is the estimation of $\boldsymbol{\xi}_{i}, \hat{\sigma}$ is the estimation of $\sigma, \lambda_{1, i}$ and $\lambda_{2, i}$ are virtual states, $\boldsymbol{\alpha}=\left[\alpha_{1}, \alpha_{2}, \alpha_{3}\right]$ and $\boldsymbol{\beta}=\left[\beta_{1}, \beta_{2}\right]$ are positive design parameters, $\varepsilon$ is the design high-gain parameter, $\hat{P}_{i}$ is the estimation of the CPL, $\hat{\boldsymbol{\eta}}_{i}$ is the estimation of $\boldsymbol{\eta}_{i}$ and $\hat{\boldsymbol{\xi}}_{2, k}$ is the estimation of $\boldsymbol{\xi}_{2, k}$.

The tuning of the parameters in this observer requires the definition of some extra matrices. Define for $i=1,2$ the following matrices

$$
\mathbf{B}_{2} \triangleq\left[\begin{array}{c}
0_{1,1} \\
1
\end{array}\right] \in \mathbb{R}^{2 \times 1}, \quad \mathbf{E}_{i} \triangleq\left[\begin{array}{cc}
-\alpha_{i} & 0 \\
-\beta_{i} & 0
\end{array}\right] \in \mathbb{R}^{2 \times 2} .
$$


Next, let $\mathbf{M}_{3} \in \mathbb{R}^{5 \times 5}$ be a matrix recursively constructed as follows

$$
\begin{aligned}
& \mathbf{M}_{1} \triangleq \mathbf{E}_{1}, \quad \mathbf{M}_{2} \triangleq\left[\begin{array}{cc}
\mathbf{M}_{1} & \mathbf{B}_{2} \mathbf{B}_{2}^{\top} \\
{\left[\begin{array}{c}
\alpha_{2} \\
\beta_{2}
\end{array}\right] \mathbf{B}_{2}^{\top}} & \mathbf{E}_{2}
\end{array}\right] \\
& \mathbf{M}_{3} \triangleq\left[\begin{array}{cc}
\mathbf{M}_{2} & 0 \\
\alpha_{3} \mathbf{B}_{4}^{\top} & -\alpha_{3}
\end{array}\right] .
\end{aligned}
$$

Lemma III.2. [37] Consider a matrix $\boldsymbol{P}_{h g o}=\boldsymbol{P}_{h g o}^{\top}>0$ and $q>0$ that satisfy the following

$$
\boldsymbol{P}_{h g o} \boldsymbol{M}_{3}+\boldsymbol{M}_{3}^{\top} \boldsymbol{P}_{h g o} \leq-q \boldsymbol{I}
$$

Then, there exists a positive value $\varepsilon_{1}^{*}$ such that for all $\varepsilon \leq$ $\min \left\{\varepsilon_{1}^{*}, 1\right\}$ the estimation error of the observer (11) satisfies the following ultimate bounds for $q=1,2$,

$$
\begin{aligned}
\left|\xi_{q, i}-\hat{\xi}_{q, i}\right| & \leq \varepsilon^{4-q} k_{1}\left|P_{i}-\hat{P}_{i}\right|+\varepsilon^{4-q} k_{2}\left\|\boldsymbol{\eta}_{i}-\hat{\boldsymbol{\eta}}_{i}\right\| \\
& +\varepsilon^{4-q} k_{3}\left|\boldsymbol{\xi}_{2, k}-\hat{\boldsymbol{\xi}}_{2, k}\right|+\varepsilon^{4-q} k_{w}|\dot{w}| \\
|\sigma-\hat{\sigma}| & \leq \varepsilon k_{1}\left|P_{i}-\hat{P}_{i}\right|+\varepsilon k_{2}\left\|\boldsymbol{\eta}_{i}-\hat{\boldsymbol{\eta}}_{i}\right\| \\
& +\varepsilon k_{3}\left|\boldsymbol{\xi}_{2, k}-\hat{\boldsymbol{\xi}}_{2, k}\right|+\varepsilon k_{w}|\dot{w}|
\end{aligned}
$$

where $k_{1}, \ldots, k_{3}$ and $k_{w}$ are some positive constants independent from $\varepsilon$.

The implementation of the observer (11), requires the computation of $\hat{\xi}_{2, k}$. This factor is not locally estimated by the observer nor measured by any sensor, but is being computed by the observers allocated in the neighbour DGUs. Therefore, the computation of (11) requires the transmission of $\hat{\xi}_{2, i}$ between observers in neighbour DGUs.

\section{B. Estimation of the power line currents, $\boldsymbol{\eta}_{i}$}

A crucial part for the computation of (6) and the observer implementation in (11) is to achieve an accurate estimation of the power line currents, $\boldsymbol{\eta}_{i}$. The idea proposed in this work is to use the value of the virtual state $\hat{\sigma}$ to compute an auxiliary signal that allows the design of an observer for the $\boldsymbol{\eta}_{i}$ dynamics.

Assume that the extended Astolfi/Marconi observer (11) is used to estimate $\xi_{1, i}, \xi_{2, i}$ and the virtual state, $\sigma$. Moreover, notice that $\sigma=\phi_{i}\left(\boldsymbol{\xi}_{i}, u_{i}, P_{i}, \boldsymbol{\eta}_{i}\right)+w_{1}$. Then, by inspection of (9), the following holds:

$$
\begin{aligned}
\mu_{i} & \triangleq \sum_{k \in \mathscr{E}_{i}} \frac{R_{k}}{L_{k}} \eta_{k, i}+w_{1}-\Delta \phi, i=C_{i} \hat{\sigma}-\frac{1}{L_{t i}}\left(-\hat{\xi}_{1, i}+u_{i}\right) \\
& +\sum_{k \in \mathscr{E}_{i}}\left(\frac{1}{L_{k}}\left(\hat{\xi}_{1, i}-\hat{\xi}_{1, k}\right)\right)+\frac{1}{R_{i}} \hat{\xi}_{2, i}-\hat{P}_{i} \frac{\hat{\xi}_{2, i}}{\hat{\xi}_{1, i}^{2}}
\end{aligned}
$$

where $\Delta \phi, i \triangleq \phi_{i}\left(\boldsymbol{\xi}_{i}, u_{i}, P_{i}, 0\right)-\phi_{i}\left(\hat{\boldsymbol{\xi}}_{i}, u_{i}, \hat{P}_{i}, 0\right)+\sigma-\hat{\sigma}$. Now, take the value $\mu_{i}$ (15) as the measured signal of the $\boldsymbol{\eta}_{i}$ dynamics. Then, the following partially linear system is obtained:

$$
\begin{aligned}
\dot{\boldsymbol{\eta}}_{i} & =\mathbf{A}_{i} \boldsymbol{\eta}_{i}+\sum_{k \in \mathscr{E}_{i}} \frac{1}{L_{k}}\left(\xi_{1, i}-\xi_{1, k}\right)+\mathbf{I}_{m_{i}} \mathbf{w}_{2} \\
\mu_{i} & =\mathbf{C}_{i} \boldsymbol{\eta}_{i}+w_{1}-\Delta \phi, i
\end{aligned}
$$

where $\mathbf{I}_{m_{i}}$ is a $m_{i} \times m_{i}$ identity matrix,

$$
\mathbf{w}_{2} \triangleq\left[w_{2,1}, \quad \ldots, \quad w_{2, m_{i}}\right]^{\top}
$$

and $\mathbf{A}_{i} \in \mathbb{R}^{m_{i} \times m_{i}}$ and $\mathbf{C}_{i} \in \mathbb{R}^{1 \times m_{i}}$ are matrices such that

$$
\mathbf{A}_{i} \triangleq\left[\begin{array}{ccc}
-\frac{R_{1}}{L_{1}} & & 0 \\
& \ddots & \\
0 & & -\frac{R_{m_{i}}}{L_{m_{i}}}
\end{array}\right], \quad \mathbf{C}_{i}=\left[\frac{R_{1}}{L_{1}}, \ldots, \frac{R_{m_{i}}}{L_{m_{i}}}\right] .
$$

It can be seen that, the introduction of the auxiliary signal, $\mu_{i}$, has transformed the $\boldsymbol{\eta}_{i}$ dynamics into a LTI observable system with a non-linear disturbance in the sensor equation. This work proposes the implementation of a Luenberger observer, due to its simplicity in design and tuning.

\section{Lemma III.3. Consider a linear Luenberger observer}

$$
\dot{\hat{\boldsymbol{\eta}}}_{i}=\boldsymbol{A}_{i} \hat{\boldsymbol{\eta}}_{i}+\sum_{k \in \mathscr{E}_{i}} \frac{1}{L_{k}}\left(\hat{\xi}_{1, i}-\hat{\xi}_{1, k}\right)+\boldsymbol{L}_{i}\left(\mu_{i}-\boldsymbol{C}_{i} \hat{\boldsymbol{\eta}}_{i}\right)
$$

where $\mu_{i}$ is the auxiliary signal computed as (15), $\boldsymbol{L}_{i} \in \mathbb{R}^{m_{i} \times 1}$ is a design matrix such that $\boldsymbol{A}_{i}-\boldsymbol{L}_{i} \boldsymbol{C}_{i}$ has all the eigenvalues in the open left-half plane.

Then, the estimation error $\boldsymbol{\eta}_{i}-\hat{\boldsymbol{\eta}}_{i}$ is ultimately bounded as follows:

$$
\begin{aligned}
& \left\|\boldsymbol{\eta}_{i}-\hat{\boldsymbol{\eta}}_{i}\right\| \leq k_{4}\left|\boldsymbol{\xi}_{i}-\hat{\boldsymbol{\xi}}_{i}\right|+k_{5}\left\|\boldsymbol{\xi}_{1, k}-\hat{\boldsymbol{\xi}}_{1, k}\right\| \\
& \quad+k_{6}|\sigma-\hat{\sigma}|+k_{7}\left|P_{i}-\hat{P}_{i}\right|+k_{w, 2}\left|w_{1}\right|+k_{w, 3}\left\|\boldsymbol{w}_{2}\right\|
\end{aligned}
$$

where $k_{4}, \ldots, k_{6}$ and $k_{w, 2}, k_{w, 3}$ are some positive constants.

Proof. As the matrix $\mathbf{A}_{i}-\mathbf{L}_{i} \mathbf{C}_{i}$ is Hurwitz by design, there is a matrix $\mathbf{P}=\mathbf{P}^{\boldsymbol{\top}}>0$ such that:

$$
\mathbf{P}\left(\mathbf{A}_{i}-\mathbf{L}_{i} \mathbf{C}_{i}\right)+\left(\mathbf{A}_{i}-\mathbf{L}_{i} \mathbf{C}_{i}\right)^{\top} \mathbf{P}=-\mathbf{Q},
$$

where $\mathbf{Q}$ is positive defined matrix.

Consider the error dynamics between (17) and (16), $\mathbf{e}_{\eta} \triangleq$ $\boldsymbol{\eta}_{i}-\hat{\boldsymbol{\eta}}_{i}$, and consider the radially unbounded Lyapunov candidate $V=\mathbf{e}_{\eta}^{\top} \mathbf{P} \mathbf{e}_{\eta}$. The derivative of the function is given by:

$$
\begin{aligned}
\dot{V} & =-\mathbf{e}_{\eta}^{\top} \mathbf{Q} \mathbf{e}_{\eta}+2 \mathbf{e}_{\eta}^{\top} \mathbf{P}\left(\xi_{1, i}-\hat{\xi}_{1, i}\right)+2 \mathbf{e}_{\eta}^{\top} \mathbf{P}\left(\boldsymbol{\xi}_{1, k}-\hat{\boldsymbol{\xi}}_{1, k}\right) \\
& -2 \mathbf{e}_{\eta}^{\top} \mathbf{P} \mathbf{L}_{i} w_{1}+2 \mathbf{e}_{\eta}^{\top} \mathbf{P} \mathbf{L}_{i} \Delta \phi, i-2 \mathbf{e}_{\eta}^{\top} \mathbf{P} \mathbf{w}_{2} .
\end{aligned}
$$

As the function $\phi_{i}\left(\boldsymbol{\xi}_{i}, u_{i}, P_{i}, 0\right)$ is (locally) Lipschitz, there are some positive constants $L_{1}, L_{2}, L_{3}$ and $L_{4}$ such that

$$
|\Delta \phi, i| \leq L_{1}\left\|\boldsymbol{\xi}_{i}-\hat{\boldsymbol{\xi}}_{i}\right\|+L_{2}\left\|\boldsymbol{\xi}_{1, k}-\hat{\boldsymbol{\xi}}_{1, k}\right\|+L_{3}|\sigma-\hat{\sigma}|+L_{4}\left|P_{i}-\hat{P}_{i}\right| .
$$

Therefore, the derivative of Lyapunov function candidate is upper bounded by:

$$
\begin{aligned}
\dot{V} & \leq-\lambda_{\min }(\mathbf{Q})\left\|\mathbf{e}_{\eta}\right\|+2\left\|\mathbf{e}_{\eta}\right\|\|\mathbf{P}\|\left(\mathbf{I}_{m_{i}}+\mathbf{L}_{i} L_{1}\right)\left\|\boldsymbol{\xi}_{i}-\hat{\boldsymbol{\xi}}_{i}\right\| \\
& +2\left\|\mathbf{e}_{\eta}\right\|\|\mathbf{P}\|\left(\mathbf{I}_{m_{i}}+\mathbf{L}_{i} L_{2}\right)\left\|\boldsymbol{\xi}_{1, k}-\hat{\boldsymbol{\xi}}_{1, k}\right\| \\
& +2\left\|\mathbf{e}_{\eta}\right\|\|\mathbf{P}\|\left(\mathbf{I}_{m_{i}}+\mathbf{L}_{i} L_{3}\right)|\sigma-\hat{\sigma}| \\
& +2\left\|\mathbf{e}_{\eta}\right\|\|\mathbf{P}\|\left(\mathbf{I}_{m_{i}}+\mathbf{L}_{i} L_{4}\right)\left|P_{i}-\hat{P}_{i}\right| \\
& +2\left\|\mathbf{e}_{\eta}\right\|\left\|\mathbf{P} \mathbf{L}_{i}\right\|\left|w_{1}\right|+2\left\|\mathbf{e}_{\eta}\right\|\|\mathbf{P}\|\left\|\mathbf{w}_{2}\right\|
\end{aligned}
$$

where $\lambda_{\min }(\cdot)$ depicts the minimum eigenvalue.

The inequality in (20) shows that the system is input-to-state stable (ISS) [42] taking $\boldsymbol{\xi}_{i}-\hat{\boldsymbol{\xi}}_{i}, \boldsymbol{\xi}_{1, k}-\hat{\boldsymbol{\xi}}_{1, k}, \sigma-\hat{\sigma}, P_{i}-\hat{P}_{i}, w_{1}$ and $\mathbf{w}_{2}$ as inputs. This fact proofs the existence of the bound (18). 
Notice that, similar to the Astolfi/Marconi observer (11), the implementation of the linear observer (17), requires the transmission of $\hat{\xi}_{1, k}$ between neighbour observers.

\section{Estimation of $P_{i}$}

The computation of the DGU current estimation (6) and the auxiliary signal (15), requires an estimation of the CPL, $P_{i}$. A common approach in such situation is to include the unknown parameter as a new state of the system [22], i.e. include a state $\xi_{3, i}$ in system (8) as follows:

$$
\begin{aligned}
& \dot{\xi}_{1, i}=\xi_{2, i} \\
& \dot{\xi}_{2, i}=\phi_{i}\left(\boldsymbol{\xi}_{i}, u_{i}, \xi_{3, i}, \boldsymbol{\eta}_{i}\right) \\
& \dot{\xi}_{3, i}=0,
\end{aligned}
$$

and design an observer that can estimate $\xi_{1, i}, \xi_{2, i}$ and $\xi_{3, i}$. Nevertheless, in the concerned problem, this approach is highly sensitive to measurement noise. For this reason, it is interesting to implement an alternative parameter-estimation algorithm. This work proposes implementing a technique based on the Immersion and Invariance (I\&I) framework [39], which offers some gain margin with respect to uncertainties in the regressor vector and its stability can be proved through an $L_{2}$ integrability condition that is generically satisfied in the considered system.

Lemma III.4. Consider system (8), let $\hat{\boldsymbol{\xi}}_{i}$ be a state-estimation generated by a Astolf/Marconi observer (11) that satisfies (13) and let $\hat{\boldsymbol{\eta}}_{i}$ be a power line current estimation that satisfies (18). Define the following vector functions

$$
\begin{gathered}
f_{0, i}\left(\hat{\boldsymbol{\xi}}_{i}, \hat{q}_{i}, \hat{\boldsymbol{\eta}}_{i}, \hat{\boldsymbol{\xi}}_{1, k}\right)=\left[\begin{array}{c}
\hat{\xi}_{2, i} \\
\phi_{i}\left(\hat{\boldsymbol{\xi}}_{i}, \hat{q}_{i}, \hat{P}_{i}, \hat{\boldsymbol{\eta}}_{i}\right)
\end{array}\right], \\
f_{1, i}\left(\hat{\boldsymbol{\xi}}_{i}\right)=\left[\begin{array}{c}
0 \\
\frac{\hat{\xi}_{2, i}}{C_{i} \hat{\xi}_{1, i}^{2}}+\frac{K_{p I}}{C L_{t i} \hat{\xi}_{1, i}}
\end{array}\right]
\end{gathered}
$$

where $\hat{q}_{i}$ is the estimation of $q_{i}$, which is defined as

$$
\begin{aligned}
q_{i} & =K_{p I}\left(C_{i} \dot{V}_{i}+\sum_{k \in \mathscr{E}_{i}} I_{k, i}+\frac{1}{R_{i}} V_{i}-I_{r e f, i}\right) \\
& +K_{i I} \int\left(y_{1, i}-I_{\text {ref }, i}\right) .
\end{aligned}
$$

and $y_{1, i}$ is the measured local current $I_{t i}$.

Define the following function

$$
\beta_{i}\left(\hat{\boldsymbol{\xi}}_{i}\right)=\gamma\left(\frac{\hat{\xi}_{2, i}^{2}}{2 C_{i} \hat{\xi}_{1, i}^{2}}+\frac{K_{p I} \hat{\xi}_{2, i}}{C_{i} L_{t i} \hat{\xi}_{1, i}}\right)
$$

where $\gamma$ is a positive constant to be tuned.

Moreover, consider the following parameter-estimation $d y$ namics:

$\dot{\hat{\theta}}_{i}=-\frac{\partial \beta_{i}\left(\hat{\boldsymbol{\xi}}_{i}\right)}{\partial \boldsymbol{\xi}_{i}}\left[f_{0, i}\left(\hat{\boldsymbol{\xi}}_{i}, \hat{q}_{i}, \hat{\boldsymbol{\eta}}_{i}, \hat{\boldsymbol{\xi}}_{1, k}\right)+f_{1, i}\left(\hat{\boldsymbol{\xi}}_{i}\right)\left(\hat{\theta}_{i}+\beta_{i}\left(\hat{\boldsymbol{\xi}}_{i}\right)\right)\right]$

$\hat{P}_{i}=\hat{\theta}+\beta_{i}\left(\hat{\boldsymbol{\xi}}_{i}\right)$.

Then, the following ultimate bound holds

$$
\begin{aligned}
\left|P_{i}-\hat{P}_{i}\right| & \leq k_{8}\left\|\boldsymbol{\xi}_{i}-\hat{\boldsymbol{\xi}}_{i}\right\|+k_{9}\left\|\boldsymbol{\xi}_{1, k}-\hat{\boldsymbol{\xi}}_{1, k}\right\| \\
& +k_{10}\left\|\boldsymbol{\eta}_{i}-\hat{\boldsymbol{\eta}}_{i}\right\|+k_{w, 3}\left|w_{1}\right|
\end{aligned}
$$

where $k_{8}, . ., k_{10}$ and $k_{w, 3}$ are some positive constants.

Proof. Define the off-the-manifold variable

$$
z \triangleq \hat{\theta}-P_{i}+\beta_{i}\left(\boldsymbol{\xi}_{i}\right) .
$$

Then, the dynamics of the off-the-manifold coordinates $z$ are given by:

$$
\dot{z}=-\gamma\left(\frac{\xi_{2, i}}{C_{i} \xi_{1, i}^{2}}+\frac{K_{p I}}{C L_{t i} \xi_{1, i}}\right)^{2} z+\delta
$$

where $\delta$ is defined as:

$$
\begin{aligned}
\delta & \triangleq \frac{\partial \beta_{i}\left(\boldsymbol{\xi}_{i}\right)}{\partial \boldsymbol{\xi}_{i}}\left[f_{0, i}\left(\boldsymbol{\xi}_{i}, q_{i}, \boldsymbol{\eta}_{i}, \boldsymbol{\xi}_{1, k}\right)+f_{1, i}\left(\boldsymbol{\xi}_{i}\right)\left(\hat{\theta}_{i}+\beta_{i}\left(\boldsymbol{\xi}_{i}\right)\right)\right. \\
& \left.+w_{1}\right]-\frac{\partial \beta_{i}\left(\hat{\boldsymbol{\xi}}_{i}\right)}{\partial \boldsymbol{\xi}_{i}}\left[f_{0, i}\left(\hat{\boldsymbol{\xi}}_{i}, \hat{q}_{i}, \hat{\boldsymbol{\eta}}_{i}\right)+\right. \\
& \left.+f_{1, i}\left(\hat{\boldsymbol{\xi}}_{i}\right)\left(\hat{\theta}_{i}+\beta_{i}\left(\hat{\boldsymbol{\xi}}_{i}\right)\right)\right] .
\end{aligned}
$$

The functions $\beta_{i}, f_{0, i}, f_{1, i}$ and $\frac{\partial \beta_{i}\left(\boldsymbol{\xi}_{i}\right)}{\partial \boldsymbol{\xi}_{i}}$ are (locally) Lipschitz. Moreover, the factor $\frac{\partial \beta_{i}\left(\boldsymbol{\xi}_{i}\right)}{\partial \boldsymbol{\xi}_{i}}$ is upper bounded. Therefore, there exist some positive constants $L_{\delta, 1}, L_{\delta, 2}, L_{\delta, 3}$ and $\beta_{\max }$ such that

$$
\begin{aligned}
\|\delta\| & \leq L_{\delta, 1}\left\|\boldsymbol{\xi}_{i}-\hat{\boldsymbol{\xi}}_{i}\right\|+L_{\delta, 2}\left\|\boldsymbol{\eta}_{i}-\hat{\boldsymbol{\eta}}_{i}\right\|+L_{\delta, 3}\left\|\boldsymbol{\xi}_{1, k}-\hat{\boldsymbol{\xi}}_{1, k}\right\| \\
& +\beta_{\text {max }}\left|w_{1}\right| .
\end{aligned}
$$

Consider the Lyapunov function candidate:

$$
V=\frac{1}{2}(z)^{2}
$$

The derivative of (26) satisfies the following

$$
\begin{aligned}
\dot{V} & =-z \gamma\left(\frac{\xi_{2, i}}{C_{i} \xi_{1, i}^{2}}+\frac{K_{p I}}{C L_{t i} \xi_{1, i}}\right)^{2} z+z \delta \\
& \leq-z \gamma\left(\frac{\xi_{2, i}}{C_{i} \xi_{1, i}^{2}}+\frac{K_{p I}}{C L_{t i} \xi_{1, i}}\right)^{2} z+z L_{\delta, 1}\left\|\boldsymbol{\xi}_{i}-\hat{\boldsymbol{\xi}}_{i}\right\| \\
& +z L_{\delta, 2}\left\|\boldsymbol{\eta}_{i}-\hat{\boldsymbol{\eta}}_{i}\right\|+z L_{\delta, 3}\left\|\boldsymbol{\xi}_{1, k}-\hat{\boldsymbol{\xi}}_{1, k}\right\| \\
& +z \beta_{\max }\left|w_{1}\right|
\end{aligned}
$$

Generically, the variable $\hat{\xi}_{1, i}$ is upper and lower bounded. Thus, the factor $\frac{\hat{\xi}_{2, i}}{C_{i} \hat{\xi}_{1, i}^{2}}+\frac{K_{p I}}{C L_{t i} \hat{\xi}_{1, i}}$ is not $L_{2}$ integrable. Therefore, by inspection of (27), it is possible to show that (26) is a ISS-Lyapunov function with linear ISS-gain [42] from $\boldsymbol{\xi}-\hat{\boldsymbol{\xi}}, \boldsymbol{\eta}_{i}-\hat{\boldsymbol{\eta}}_{i}$ and $\boldsymbol{\xi}_{1, k}-\hat{\boldsymbol{\xi}}_{1, k}$ to $z$. As a consequence, taking into account the relation (24) and the fact that $\beta_{i}$ is Lipschitz, the bound (23) can be deduced.

\section{Observer stability}

The last subsections have presented the sub-systems of the proposed estimation algorithm in a single DGU. The observer at each DGU can be seen as an interconnection of three estimation algorithms as it is depicted in Fig. 4. The crucial step is to study under which conditions the coupling between the three 
estimation algorithms creates a stable structure. After that, it is necessary to study the conditions in which the interconnection of FDIA estimation algorithms is stable for an arbitrary DC microgrid topology.

In order to proof the stability of the observer, it is of interest to define the following vector

$$
\chi_{i} \triangleq\left[\begin{array}{c}
\xi_{1, i}-\hat{\xi}_{1, i} \\
\xi_{2, i}-\hat{\xi}_{2, i} \\
\sigma-\hat{\sigma}
\end{array}\right] .
$$

Lemma III.5. Consider the case without uncertainty/disturbances, i.e. $w_{1}=0$ and $w_{2, j}=0$ for $j=$ $1, \ldots, m_{i}$. Moreover, consider an observer composed by (11), which satisfies the bound (13), the linear observer (17) that satisfies (18) and the parameter-estimation (22) that satisfies (23). Then, if the following condition holds,

$$
k_{7} k_{10}<1
$$

there is a value $\varepsilon_{2}^{*}$ such that, for all $\varepsilon \leq \varepsilon_{2}^{*}$, the variable $\chi_{i}$ is ultimately bounded as follows,

$$
\left\|\boldsymbol{\chi}_{i}\right\| \leq \varepsilon k_{11}\left\|\boldsymbol{\xi}_{1, k}-\hat{\boldsymbol{\xi}}_{1, k}\right\|+\varepsilon k_{12}\left\|\boldsymbol{\xi}_{2, k}-\hat{\boldsymbol{\xi}}_{2, k}\right\| .
$$

where $k_{11}$ and $k_{12}$ are some positive constants.

Proof. Taking into account (13) and the definition of $\chi_{i}$, there are some positive constant $n_{1}$ and $n_{2}$, such that the following bounds hold

$$
\begin{aligned}
\left\|\boldsymbol{\chi}_{i}\right\| & \leq \varepsilon \sqrt{n_{1}} k_{1}\left|P_{i}-\hat{P}_{i}\right|+\varepsilon \sqrt{n_{1}} k_{2}\left\|\boldsymbol{\eta}_{i}-\hat{\boldsymbol{\eta}}_{i}\right\| \\
& +\varepsilon \sqrt{n_{1}} k_{3}\left|\boldsymbol{\xi}_{2, k}-\hat{\boldsymbol{\xi}}_{2, k}\right| .
\end{aligned}
$$

Consider (18), (23), the second equation of (30) and assume that $k_{7} k_{10}<1$. Then, the following bound is obtained:

$$
\begin{aligned}
\left\|\chi_{i}\right\| & \leq \varepsilon \sqrt{n_{1}}\left(\frac{k_{1} k_{8}+k_{1} k_{10} \max \left\{k_{4}, k_{6}\right\}}{1-k_{10} k_{7}}+k_{2} \max \left\{k_{4}, k_{6}\right\}\right. \\
& \left.+\frac{k_{2} k_{7} k_{8}+k_{2} k_{7} k_{10} \max \left\{k_{4}, k_{6}\right\}}{1-k_{10} k_{7}}\right)\left\|\boldsymbol{\chi}_{\boldsymbol{i}}\right\| \\
& +\varepsilon \sqrt{n_{1}}\left(\frac{k_{1} k_{9}+k_{1} k_{10} k_{5}}{1-k_{10} k_{7}}\right. \\
& \left.+k_{2} k_{5} \frac{k_{2} k_{7} k_{9}+k_{2} k_{7} k_{10} k_{5}}{1-k_{10} k_{7}}\right)\left\|\boldsymbol{\xi}_{1, k}-\hat{\boldsymbol{\xi}}_{1, k}\right\| \\
& +\varepsilon \sqrt{n_{1}} k_{3}\left\|\boldsymbol{\xi}_{2, k}-\hat{\boldsymbol{\xi}}_{2, k}\right\| .
\end{aligned}
$$

Notice that the bound in (31) reduces with $\varepsilon$. Therefore, there is a value $\varepsilon_{2}^{*}$ such that for $\varepsilon \leq \varepsilon_{2}^{*}$, the bound (31) reduces to the ultimate bound (29).

The existence of the ultimate bound (29), shows that, the local estimation structure at each DGU is ISS from the estimation error of the neighbouring observers if condition (28) is satisfied. This fact can be used to present a condition for the stability of an interconnection of observers for an arbitrary communication graph of the DC microgrid. Taking into account (29), the following ultimate bound for the $i_{t h}$ DGU can be deduced

$$
\left\|\chi_{i}\right\| \leq \varepsilon \max \left\{k_{11}, k_{12}\right\} \sum_{k \in \mathscr{E}_{i}}\left\|\chi_{k}\right\| .
$$

Theorem III.1. Consider a distributed observer that satisfies (32). Then, there are a set of positive constants $\varepsilon_{3, i}^{*}$ for $i=$ $1, \ldots, N$, such that, for all $\varepsilon_{i} \leq \varepsilon_{3, i}^{*}$ for $i=1, \ldots, N$, the estimations $\hat{\xi}_{1, i}, \hat{\xi}_{2, i}, \hat{P}_{i}$ and $\boldsymbol{\eta}_{i}$ converges to its true value.

Proof. As the observer in the $i_{t h}$ DGU satisfies the bound (32), the $\chi_{i}$ dynamics are ISS from $\chi_{k}$ for $k=1, \ldots, m_{i}$. Define $\gamma_{i, j}$ as the ISS gain from $\chi_{j}$ to $\chi_{i}$. As the communication graph of the microgrid is connected and without self-loops, and all the ISS gains are linear, the small-gain theorem reduces to a set of function compositions of $\gamma_{i, j}$ and $\left(1-\gamma_{i, j}\right)^{-1}$ that have to be smaller than 1 (define a contraction) to ensure the estimation convergence [42]. The ISS gains, $\gamma_{i, j}$ are proportional to the design parameter $\varepsilon_{i}$. Therefore, the functions $\gamma_{i, j}$ and $(1-$ $\left.\gamma_{i, j}\right)^{-1}$ and its possible compositions can be made arbitrary small by reducing $\varepsilon_{i}$ for $i=1, \ldots, N$, which proves the theorem.

\section{Numerical Simulations}

This section aims to study the behaviour of the detection and mitigation algorithm in a set of numerical simulations that consider significant sensor noise and model uncertainty.

\section{A. Microgrid with 4 agents}

A DC microgrid composed of 4 DGUs interconnected, as depicted in Fig. 5, is considered in the Simulation. Each DGU has an unknown CPL connected at its point of coupling to the microgrid.

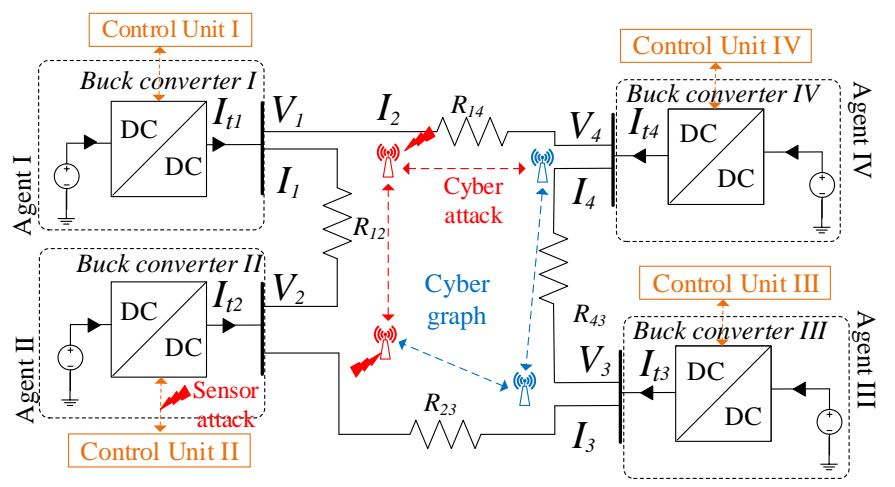

Fig. 5. Topology of the considered DC microgrid with 4 DGUs. Blue arrows represent the cyber-layer and black lines depict the physical circuit.

The whole microgrid is controlled to ensure the convergence of the average voltage to $315 \mathrm{~V}$. During the simulation, there is a set of DGU output current cyber-link FDIA attacks. Specifically, at time $t=4 \mathrm{~s}$ there is a FDIA in the cyberlinks that connect the DGU 1 with its neighbours, and at time $t=4.5 \mathrm{~s}$ there is a simultaneous attack in the cyber-links of the DGU 2 and the cyber-links of DGU 4. The FDIA signal in the DGU 1 consists of a step function of value $8 \mathrm{~A}$. In the DGU 2, the FDIA signal consists of a step function of value $3 A$. Finally, the FDIA signal in DGU 4 consists of a sinusoidal of amplitude $0.5 \mathrm{~A}$, bias $2 \mathrm{~A}$ and frequency $10 \mathrm{rad} / \mathrm{s}$.

The presence of these attacks does not destabilize the microgrid nor prevents the convergence of the average voltage, 


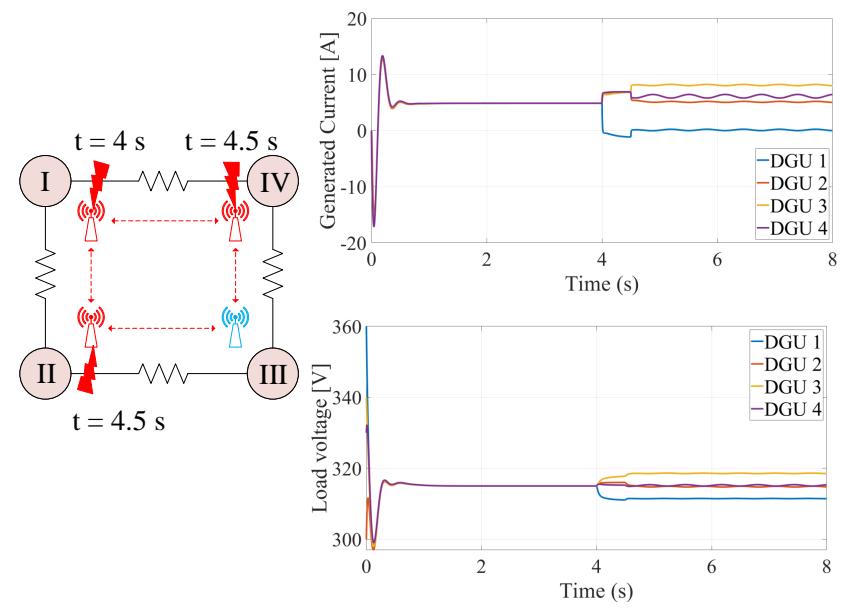

Fig. 6. Current and voltage evolution under FDIAs. At $t=4 \mathrm{~s}$ there is a FDIA in the DGU 1 cyber-links and at time $t=4.5 \mathrm{~s}$ there is a simultaneous attack in the cyber-links of the DGU 2 and the cyber-links of DGU 4.

this fact can be observed in Fig. 6. However, it significantly modifies the behaviour of the microgrid and prevents the equal current sharing between agents.

It is considered that the DC microgrid parameters are not accurate, which is translated to some parametric uncertainty. In Table II, it is depicted the true DGU and power line parameter values and the ones used in the observer equations. The only parameters that are assumed to be known exactly are the $P I$ controller design parameters, which are fixed with $K_{p I}=29.99$ and $K_{i I}=168.1$, in each DGU.

TABLE II

TRUE DGU PARAMETERS AND MODEL PARAMETER VALUES USED IN THE OBSERVERS.

\begin{tabular}{|c|c|c|}
\hline Symbol & True Value & Model value \\
\hline$L_{t i}$ & $1[H]$ & $0.8[H]$ \\
$C_{i}$ & $0.05[F]$ & $0.055[F]$ \\
$R_{i}$ & $96[\Omega]$ & $90[\Omega]$ \\
$R_{12}, R_{23}$ & $1.8[\Omega]$ & $1[\Omega]$ \\
$R_{14}, R_{34}$ & $1.3[\Omega]$ & $1.8[\Omega]$ \\
$L_{12}, L_{23}$ & $50 \cdot 10^{-6}[H]$ & $30 \cdot 10^{-6}[H]$ \\
$L_{14}, L_{34}$ & $50 \cdot 10^{-6}[H]$ & $60 \cdot 10^{-6}[H]$ \\
$P_{i}$ & $500[W]$ & $-[W]$ \\
\hline
\end{tabular}

Moreover, it is assumed that the voltage and current sensors are corrupted by some high-frequency Gaussian noise. Specifically, the voltage sensor, $V_{1}$, and the voltage signals transmitted between DGUs are affected by random noise with variance 0.0102 . The current sensor is affected with Gaussian noise of variance 0.001 .

As the DGUs are assumed to be identical, the observer design parameters are tuned identically in each DGU following the next methodology, which has been motivated by the theory provided in the last section.

1) The value of $\gamma$ has been fixed at an arbitrary positive value 2 .

2) The Luenberger observer gains, $L_{i}$, have been tuned to minimize the $H_{\infty}$ norm between the estimation error and

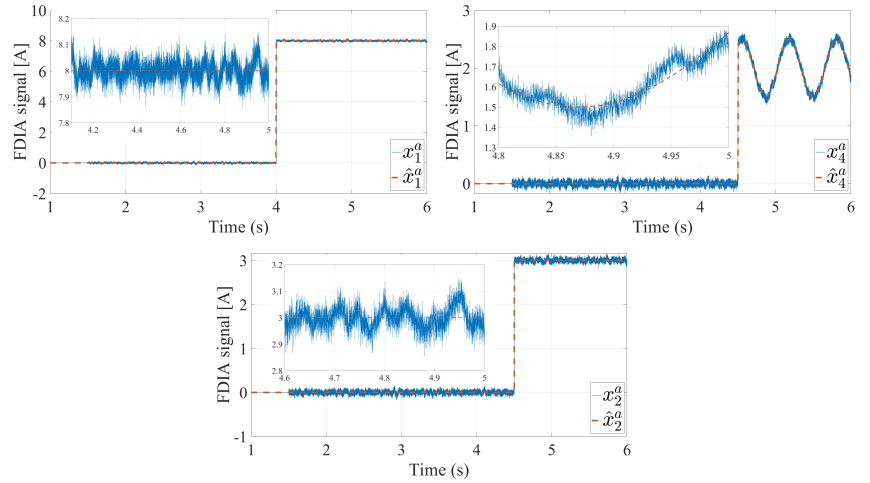

Fig. 7. Evolution of the attack estimation and true attack signal value in the compromised DGUs. The estimation is initially zero due to the warm-up time of $1.5 \mathrm{~s}$.

the output disturbances, as explained in ([43], Chapter 9). This is sufficient to satisfy conditions (19) and (28).

3) The parameters $\alpha_{1}, \alpha_{2}, \alpha_{3}, \beta_{1}$ and $\beta_{2}$ have been fixed through the algorithm in [44]. This is sufficient to satisfy the condition (13).

4) The high-gain parameter $\varepsilon$ has been decreased until adequate performance is obtained.

The value of said parameters is summarized in Table III.

TABLE III

OBSERVER DESIGN PARAMETERS.

\begin{tabular}{|c|c|c|c|}
\hline Parameter & Value & Parameter & Value \\
\hline$\alpha_{1}$ & 3 & $L_{2}$ & 2.5997 \\
$\alpha_{2}$ & 3 & $\beta_{1}$ & 4.222 \\
$\alpha_{3}$ & 1 & $\beta_{2}$ & 1.0526 \\
$\gamma$ & 2 & $\varepsilon$ & 0.02 \\
$L_{1}$ & -3.5997 & & \\
\hline
\end{tabular}

This simulation considers the case scenario in which no prior information of the power line currents or the local power load.

The evolution of the FDIAs signals and the observer reconstruction is depicted in Fig. 7. Naturally, the presence of sensor noise does not destabilize the observer, but prevents the convergence of the estimation to zero. The estimation error presents a variance of approximately 0.0012 , which is coherent with the considered current sensor noise. Nevertheless, as it will be shown later, the current attack reconstruction accuracy is sufficient in order to get an adequate attack mitigation. Additionally, it can be noticed that, even in the presence of significant model uncertainty, the bias of the attack estimation is minimal. These results validate the robustness and scalability of the proposed estimation algorithm.

The reconstructed attacks have been used to mitigate the FDIAs following the idea presented in (5). The behaviour of the DC microgrid current and voltage under the proposed attack mitigation strategy is depicted in Fig. 8. By comparing the pre-attack and post-attack system behaviour, it can be seen that even in the presence of significant sensor noise and model uncertainty, the proposed strategy is capable of 


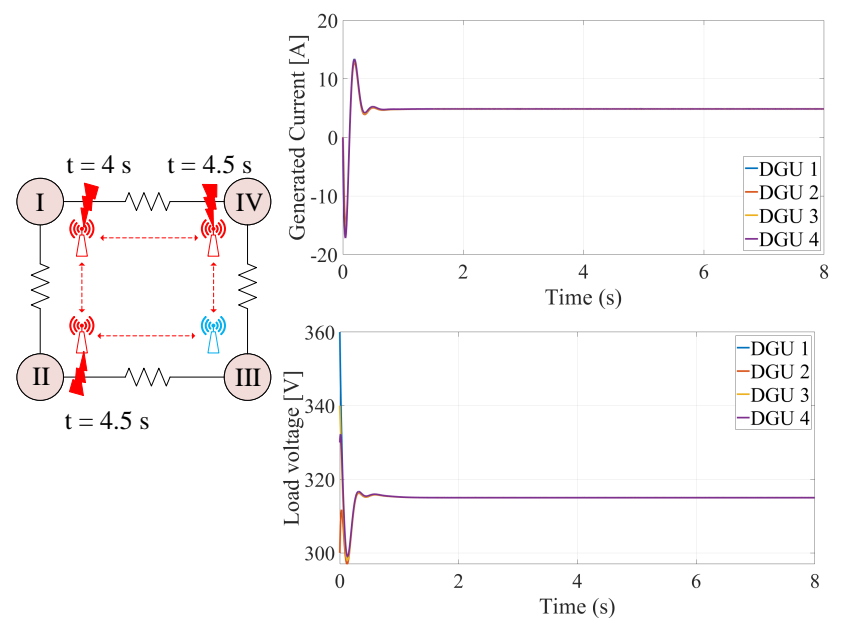

Fig. 8. Current and voltage evolution under FDIAs and observer reconstruction and mitigation. At $t=4 \mathrm{~s}$ there is a FDIA in the DGU 1 cyber-links. At $t=4.5 \mathrm{~s}$ there is a FDIA in the DGU 2 and 4 cyber-links.

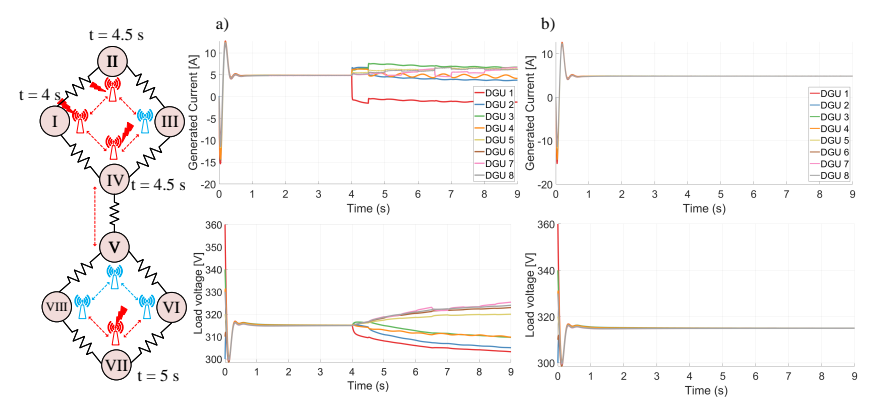

Fig. 9. Current and voltage evolution under FDIAs. At $t=4 \mathrm{~s}$ there is a FDIA in the DGU 1 cyber-links and at time $t=4.5 \mathrm{~s}$ there is a simultaneous attack in the cyber-links of the DGU 2 and the cyber-links of DGU 4, and at $t=5 s$ there is an attack in the Cyber-links of the DGU 7. In the first case scenario, a), the system is not protected. In the second case scenario, b), the system is protected by the proposed observer-based approach.

mitigating immediately the effect of FDIAs on the system with insignificant effects on the system performance.

\section{B. Microgrid with 8 agents}

In order to validate the scalability of the proposed approach, a second simulation has been performed with a DC microgrid composed by 8 DGUs. Each DGU has an unknown CPL connected at its point of coupling and the topology of the grid is depicted in the left-hand side of Fig. 9.

Similar to the last simulation, the whole microgrid is controlled to ensure the convergence of the average voltage to $315 \mathrm{~V}$. During the simulation, there is a set of DGU output current cyber-link FDIA attacks. Specifically, DGU 1, 2 and 4 are compromised with the same FDIA value as in the last simulation scenario. Additionally, at time $t=5 \mathrm{~s}$, there is a FDIA in the cyber-links of the DGU 7 that consists of random steps of duration $0.5 \mathrm{~s}$. The exact value of the attack signal is $x_{7}^{a}=[1,0.2,-0.5,2,0.5]$.

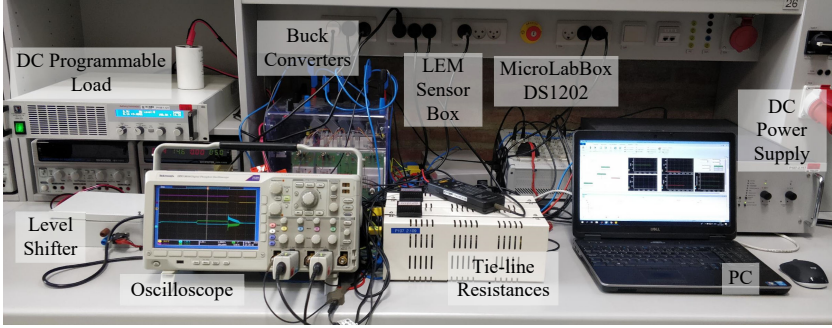

Fig. 10. Experimental setup of a cooperative DC microgrid comprising of $N$ $=2$ agents controlled by dSPACE MicroLabBox DS1202 supplying power to the programmable CPL.

It can be observed in the case scenario a) of Fig. 9 that the average voltage still satisfies the instantaneous objective after the cyber-attack, but the load voltage of each DGU presents an unstable behaviour. This is due to the interaction between the CPL and the FDIA. The objective is to implement the proposed technique in order to detect the presence of FDIA and mitigate its effect in order to preserve the stability of the system.

Again, it is considered that the DC microgrid parameters are not accurate, and the sensors are corrupted with high-frequency noise. The values of the uncertainty and noise are of the same order as in the last simulation. Due to space restrictions, the exact value of the parameters have been obviated.

The behaviour of the DC microgrid current and voltage under the proposed attack mitigation strategy is depicted in the second case scenario b) of Fig. 9. By comparing the pre-attack and post-attack system behaviour, it can be seen that even in the presence of significant sensor noise and model uncertainty and FDIA with random step values, the proposed strategy is capable of mitigating immediately the effect of FDIAs on the system with insignificant effects on the system performance.

\section{EXPERIMENTAL VALIDATION}

The proposed mitigation strategy has been validated in an experimental prototype of DC microgrid operating at a voltage reference $V_{d c_{r e f}}$ of $48 \mathrm{~V}$ with $N=2$ DC-DC buck converters. An image of the experimental setup is depicted in Fig. 10(a). Both converters are tied radially via tie-line resistances in a physical ring-bus network with a programmable load, where a constant value of demand can be programmed in one of the buses. Each converter is controlled by dSPACE MicroLabBox DS1202 (target), with control commands from the dSPACE ControlDesk from the PC (host). The considered system consists of two sources with the converters rated equally for $600 \mathrm{~W}$. It should be noted that the controller gains are equivalent for each converter. The experimental testbed parameters and controller parameters are provided in Table IV.

Using the local and neighboring measurements, the proposed observer-based strategy is implemented in every converter (as shown in Fig. 11) to mitigate FDIAs and meet the desired control objectives in DC microgrids. The observer design parameters have been tuned following the same process as in the numerical simulation in Section IV. The resulting parameters are included in Table V. 


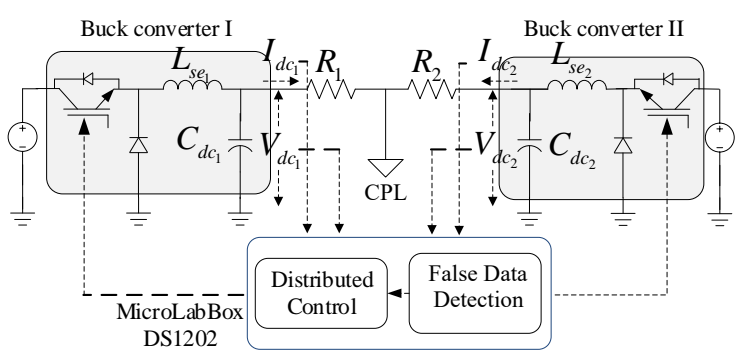

Fig. 11. Single line diagram of the experimental setup shown in Fig. 10.

TABLE IV

EXPERIMENTAL TESTBED PARAMETERS.

\begin{tabular}{|c|c|}
\hline Symbol & Value \\
\hline Plant & \\
\hline$L_{s e_{i}}$ & $3[\mathrm{mH}]$ \\
$C_{d c_{i}}$ & $100[\mu \mathrm{F}]$ \\
$R_{1}$ & $0.8[\Omega]$ \\
$R_{2}$ & $1.4[\Omega]$ \\
\hline Controller & \\
\hline$V_{d c_{r e f}}$ & $48[\mathrm{~V}]$ \\
$K_{P}^{H_{1}}$ & $1.92[-]$ \\
$K_{I_{1}}^{H_{1}}$ & $15[-]$ \\
$K_{P}^{H_{2}}$ & $4.5[-]$ \\
$K_{I}^{H_{2}}$ & $0.08[-]$ \\
$g$ & $0.64[-]$ \\
\hline
\end{tabular}

TABLE V

OBSERVER DESIGN PARAMETERS IN THE EXPERIMENTAL TESTBED.

\begin{tabular}{|c|c|c|c|}
\hline Parameter & Value & Parameter & Value \\
\hline$\alpha_{1}$ & 2 & $L_{2}$ & 1.032 \\
$\alpha_{2}$ & 2 & $\beta_{1}$ & 2.84 \\
$\alpha_{3}$ & 1 & $\beta_{2}$ & 1.075 \\
$\gamma$ & 2 & $\varepsilon$ & 0.075 \\
$L_{1}$ & -1.462 & & \\
\hline
\end{tabular}

The performance of the proposed algorithm has been validated in three different case scenarios where communication delay, a shift in the CPL's value and time-varying attack signals have been considered. In the first case scenario, the system is perturbed with a change of the unknown CPL and, after the CPL modification, a simultaneous cyber-attack is conducted on current measurements from both the converters with the false data, given by $I_{1}^{a}=1.5 \mathrm{~A}$ and $I_{1}^{a}=1 \mathrm{~A}$. As it can be seen in Fig. 12a), the introduction of this simultaneous cyber-attack significantly modifies the behaviour of the system and prevents consensus. In the second case scenario, first, a cyber-attack is conducted on agent I with a false data, given by $I_{1}^{a}=1.8 \mathrm{~A}$. Second, the value of the CPL is increased. Additionally, the communication channel is affected with a variable delay with a maximum value of $75 \mathrm{~ms}$. The evolution of the system under this cyber-attack is very similar as in the first case scenario in Fig. 12a) and has been obviated due to space restrictions. The purpose of this second case scenario is to study the performance of the algorithm under communication delay between observers. In the third case scenario, two cyber-attacks are conducted on DGU II with the false data modeled as a sinusoidal function $I_{2}^{a}=1.4(\sin 0.4 \pi \mathrm{t}) \mathrm{A}$ for the first event and then as a ramp function $I_{2}^{a}=1.2 \mathrm{t}$ A for the second event.

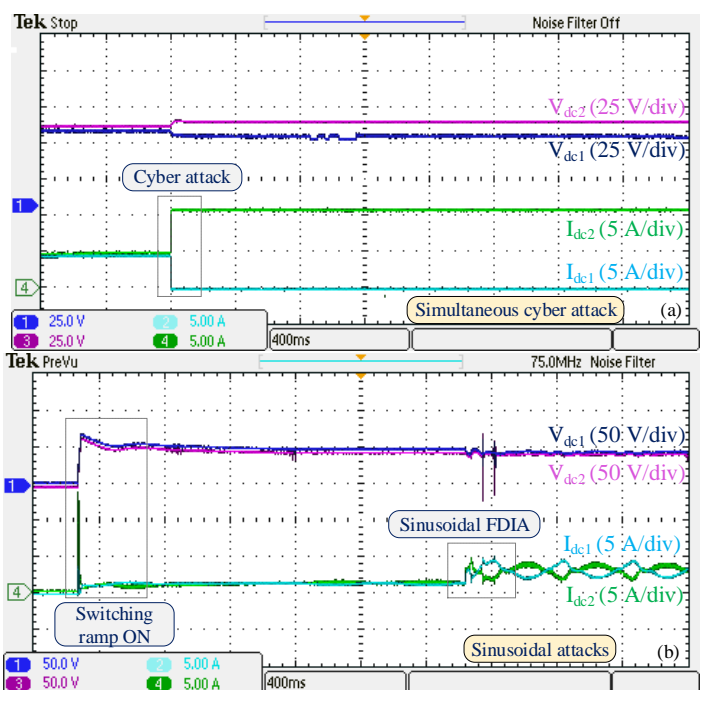

Fig. 12. Evolution of the experimental setup in the presence of FDIAs and absence of mitigation strategy: (a) simultaneous constant cyber attack on both agents, (b) ramp and sinusoidal attack element on agent II.

By comparing the pre-attack and post-attack behaviour in Fig. 12, it can be seen that the attacks modify significantly the behaviour of the system if no mitigation strategy is deployed. Alternatively, the evolution of the system in all the studied case scenarios with the proposed mitigation strategy is presented in Fig. 13. In the first case in Fig. 13(a), the response of the mitigation scheme under a change in the unknown CPL value is studied. It can be seen that the variation of CPL modifies the DGUs current set-point, but does not prevent the equal current sharing. Therefore, the modification of the CPL does not introduce a bias in the a attack estimation, which exemplifies the adaptability of the algorithm to changes on the unknown CPL value. Moreover, after the cyber-attack, the system restores back itself to the pre-attack set-points. This validates the scalability of the proposed observer strategy in providing resiliency against FDIAs.

Since a communication network is employed to transmit neighbouring estimations between observers, the validity of the proposed detection scheme needs to be studied in the presence of time delays in the communication channel. In some cases, the presence of communication delay may affect the system performance [45], thereby reducing the convergence rate and leading to sustained oscillations in the system. Furthermore, chaotic behaviour may be induced due to the interaction of the delay and the nonlinearities [46]. Fortunately, in the considered scenario, the delayed terms appear in the last equation of the extended Astolfi/Marconi observer (11) and can be modeled as a disturbance to be attenuated by the observer. It is well known that the Astolfi/Marconi observer is robust to disturbances in the last equation [37] and can attenuate its effect by reducing the design parameter $\varepsilon$. This fact can be seen in equation (13). Specifically, if there is a factor $d$ that models the disturbance induced by the communication delay, the upper-bound in (13) 


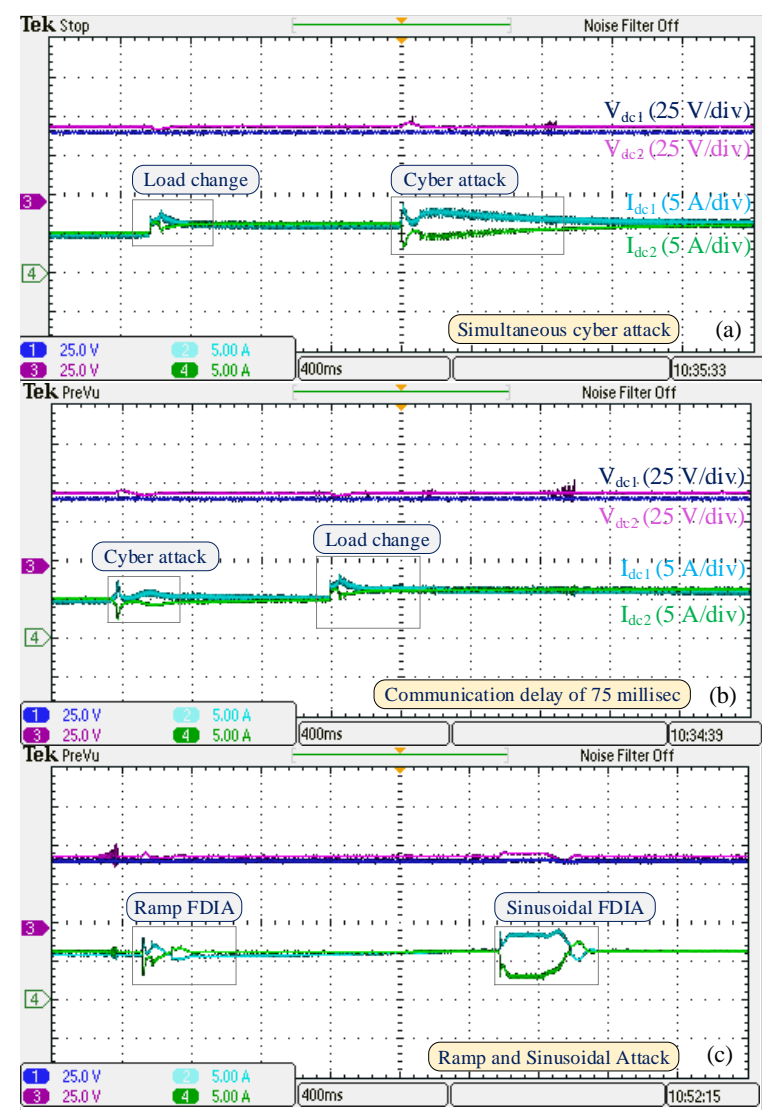

Fig. 13. Experimental validation of the proposed controller under: (a) simultaneous cyber attack on both agents, (b) cyber attack on agent I under a communication delay of $75 \mathrm{~ms}$, (c) ramp and sinusoidal attack element on agent II.

reduces to

$$
\begin{aligned}
|\sigma-\hat{\sigma}| & \leq \varepsilon k_{1}\left|P_{i}-\hat{P}_{i}\right|+\varepsilon k_{2}\left\|\boldsymbol{\eta}_{i}-\hat{\boldsymbol{\eta}}_{i}\right\| \\
& +\varepsilon k_{3}\left|\boldsymbol{\xi}_{2, k}-\hat{\boldsymbol{\xi}}_{2, k}\right|+\varepsilon k_{w}|\dot{w}|+\varepsilon k_{3}|d|,
\end{aligned}
$$

which shows that the effect of the cyber-disturbance $d$ can be diminished by reducing $\varepsilon$. Consequently, the estimator ensures resilience to delay in the communication channels. This fact has been validated in the second case study, where the performance of the algorithm under communication delay between DGUs is studied. Specifically, a variable communication delay with a maximum value of $75 \mathrm{~ms}$ is considered in the second case study in Fig. 13(b). Even though the coordination between DGUs is limited to large communication delay, it can be seen that when a FDIA is conducted under communication delays that may lead to diverging DGU output currents, the proposed observer strategy recovers pre-attack performance.

For the third case study in Fig. 13(c), two time-varying cyberattacks are conducted on DGU II. However, in the presence of the proposed mitigation strategy, it can be seen that the system restores back to the pre-attack setpoints in both types of attack. This validates the robustness of the performance of the proposed observer strategy in providing resiliency against FDIAs of time-varying nature.

\section{CONCLUSIONS}

This work presents a FDIA detection and mitigation strategy for cooperative DC microgrid's DGU current sensors and cyberlinks, even in the presence of unknown CPLs. The strategy is based on reconstructing the attack signal and cancelling its effect in the compromised measurements. It has been shown that the attack signal can be estimated by the use of a distributed observer that estimates the DGU output current accurately.

This work has presented the necessary conditions for the local stability of each individual observer and the necessary conditions for the stability of the distributed scheme. Moreover, the results have been validated in a numerical simulation and in an experimental prototype, where model uncertainty, noise, and communication delay have been considered.

\section{REFERENCES}

[1] A. Cecilia, M. Serra, and R. Costa-Castelló, "Nonlinear adaptive observation of the liquid water saturation in polymer electrolyte membrane fuel cells," J. Power Sources, vol. 492, p. 229641, 2021.

[2] A. Cecilia, J. Carroquino, V. Roda, R. Costa-Castelló, and F. Barreras, "Optimal energy management in a standalone microgrid, with photovoltaic generation, short-term storage, and hydrogen production," Energies, vol. 13, no. 6, p. 1454, Mar. 2020.

[3] T. Dragičević, X. Lu, J. C. Vasquez, and J. M. Guerrero, "Dc microgrids-part I: A review of control strategies and stabilization techniques," IEEE Trans. Power Electron., vol. 31, no. 7, pp. 4876-4891, Jul. 2016.

[4] V. Nasirian, S. Moayedi, A. Davoudi, and F. L. Lewis, "Distributed cooperative control of dc microgrids," IEEE Trans. Power Electron., vol. 30, no. 4, pp. 2288-2303, Apr. 2015.

[5] S. Sahoo and S. Mishra, "A distributed finite-time secondary average voltage regulation and current sharing controller for dc microgrids," IEEE Trans. Smart Grid, vol. 10, no. 1, pp. 282-292, Jan. 2019.

[6] M. Yazdanian and A. Mehrizi-Sani, "Distributed control techniques in microgrids," IEEE Trans. Smart Grid, vol. 5, no. 6, pp. 2901-2909, Nov. 2014.

[7] H. Sandberg, S. Amin, and K. H. Johansson, "Cyberphysical security in networked control systems: An introduction to the issue," IEEE Control Syst. Mag., vol. 35, no. 1, pp. 20-23, Feb. 2015.

[8] R. Deng, P. Zhuang, and H. Liang, "False data injection attacks against state estimation in power distribution systems," IEEE Trans. Smart Grid, vol. 10, no. 3, pp. 2871-2881, Jun. 2019.

[9] P. Danzi, M. Angjelichinoski, . Stefanović, T. Dragičević, and P. Popovski, "Software-defined microgrid control for resilience against denial-ofservice attacks," IEEE Trans. Smart Grid, vol. 10, no. 5, pp. 5258-5268, Sep. 2019

[10] F. Pasqualetti, F. Dörfler, and F. Bullo, "Attack detection and identification in cyber-physical systems," IEEE Trans. Autom. Control, vol. 58, no. 11, pp. 2715-2729, Nov. 2013.

[11] L. Liu, M. Esmalifalak, Q. Ding, V. A. Emesih, and Z. Han, "Detecting false data injection attacks on power grid by sparse optimization," IEEE Trans. Smart Grid, vol. 5, no. 2, pp. 612-621, Mar. 2014.

[12] K. Manandhar, X. Cao, F. Hu, and Y. Liu, "Detection of faults and attacks including false data injection attack in smart grid using kalman filter," IEEE Trans. Control Netw. Syst., vol. 1, no. 4, pp. 370-379, Dec. 2014.

[13] P.-Y. Chen, S. Yang, J. A. McCann, J. Lin, and X. Yang, "Detection of false data injection attacks in smart-grid systems," IEEE Commun. Mag., vol. 53, no. 2, pp. 206-213, Feb. 2015.

[14] M. Khalaf, A. Youssef, and E. El-Saadany, "Joint detection and mitigation of false data injection attacks in agc systems," IEEE Trans. Smart Grid, vol. 10, no. 5, pp. 4985-4995, 2019.

[15] A. Sargolzaei, K. Yazdani, A. Abbaspour, C. D. Crane III, and W. E. Dixon, "Detection and mitigation of false data injection attacks in networked control systems," IEEE Trans. Industr. Inform., vol. 16, no. 6, pp. 4281-4292, Jun. 2020.

[16] J. Zhao, G. Zhang, M. La Scala, Z. Y. Dong, C. Chen, and J. Wang, "Short-term state forecasting-aided method for detection of smart grid general false data injection attacks," IEEE Trans. Smart Grid, vol. 8, no. 4, pp. 1580-1590, Jul. 2017. 
[17] S. Li, Y. Yılmaz, and X. Wang, "Quickest detection of false data injection attack in wide-area smart grids," IEEE Trans. Smart Grid, vol. 6, no. 6, pp. 2725-2735, Nov. 2015.

[18] H. Nishino and H. Ishii, "Distributed detection of cyber attacks and faults for power systems," IFAC Proc. Vol., vol. 47, no. 3, pp. 11932 11937 , Aug. 2014.

[19] M. N. Kurt, Y. Yilmaz, and X. Wang, "Distributed quickest detection of cyber-attacks in smart grid," IEEE Trans. Inf. Forensics Secur., vol. 13, no. 8, pp. 2015-2030, Aug. 2018.

[20] H. M. Khalid and J. C.-H. Peng, "Immunity toward data-injection attacks using multisensor track fusion-based model prediction," IEEE Transactions on Smart Grid, vol. 8, no. 2, pp. 697-707, Mar. 2017.

[21] A. J. Gallo, M. S. Turan, F. Boem, T. Parisini, and G. Ferrari-Trecate, "A distributed cyber-attack detection scheme with application to dc microgrids," IEEE Trans. Autom. Control, vol. 65, no. 9, pp. 3800-3815, Apr. 2020.

[22] A. Cecilia, S. Sahoo, T. Dragicevic, R. Costa-Castello, and F. Blaabjerg, "Detection and mitigation of false data in cooperative dc microgrids with unknown constant power loads," IEEE Trans. Power Electron., 2021, to appear.

[23] G. Liang, J. Zhao, F. Luo, S. R. Weller, and Z. Y. Dong, "A review of false data injection attacks against modern power systems," IEEE Trans. Smart Grid, vol. 8, no. 4, pp. 1630-1638, Jul. 2017.

[24] A. S. Musleh, G. Chen, and Z. Y. Dong, "A survey on the detection algorithms for false data injection attacks in smart grids," IEEE Trans. Smart Grid, vol. 11, no. 3, pp. 2218-2234, May 2020.

[25] M. S. Sadabadi, S. Sahoo, and F. Blaabjerg, "Stability oriented design of cyber attack resilient controllers for cooperative dc microgrids," IEEE Trans. Power Electron., 2021, to appear.

[26] S. Sahoo, T. Dragičević, and F. Blaabjerg, "An event-driven resilient control strategy for dc microgrids," IEEE Trans. Power Electron., vol. 35 , no. 12, pp. 13714-13724, May 2020.

[27] — "Multilayer resilience paradigm against cyber attacks in dc microgrids," IEEE Trans. Power Electron., vol. 36, no. 3, pp. 2522 2532, Mar. 2021

[28] — "Resilient operation of heterogeneous sources in cooperative dc microgrids," IEEE Trans. Power Electron., vol. 35, no. 12, pp. $12601-$ 12605 , Apr. 2020.

[29] C. A. Soriano-Rangel, W. He, F. Mancilla-David, and R. Ortega, "Voltage regulation in buck-boost converters feeding an unknown constant power load: An adaptive passivity-based control," IEEE Trans. Control. Syst. Technol., pp. 1-8, Jan. 2020.

[30] E. Cruz-Zavala and J. A. Moreno, "Levant's arbitrary-order exact differentiator: A lyapunov approach," IEEE Trans. Autom. Control, vol. 64, no. 7, pp. 3034-3039, Oct. 2018.

[31] S. Trip, M. Cucuzzella, X. Cheng, and J. Scherpen, "Distributed averaging control for voltage regulation and current sharing in dc microgrids," IEEE Contr. Syst. Lett., vol. 3, no. 1, pp. 174-179, Jan. 2018.

[32] N. L. Diaz, T. Dragičević, J. C. Vasquez, and J. M. Guerrero, "Intelligent distributed generation and storage units for dc microgrids-a new concept on cooperative control without communications beyond droop control,' IEEE Trans. Smart Grid, vol. 5, no. 5, pp. 2476-2485, Sept. 2014.

[33] S. Sahoo, J. C. Peng, A. Devakumar, S. Mishra, and T. Dragičević, "On detection of false data in cooperative de microgrids-a discordant element approach," IEEE Trans. Ind. Electron., vol. 67, no. 8, pp. 6562-6571, Aug. 2020.

[34] S. Sahoo, S. Mishra, J. C. Peng, and T. Dragičević, "A stealth cyberattack detection strategy for dc microgrids," IEEE Trans. Power Electron., vol. 34, no. 8, pp. 8162-8174, 2019.

[35] B. Severino and K. Strunz, "Enhancing transient stability of dc microgrid by enlarging the region of attraction through nonlinear polynomial droop control," IEEE Trans. Circuits Syst. I, Reg. Papers, vol. 66, no. 11, pp. 4388-4401, Nov. 2019.

[36] R. Hermann and A. Krener, "Nonlinear controllability and observability," IEEE Trans. Autom. Control, vol. 22, no. 5, pp. 728-740, Oct. 1977.

[37] D. Astolfi and L. Marconi, "A high-gain nonlinear observer with limited gain power," IEEE Trans. Autom. Control, vol. 60, no. 11, pp. 3059-3064, Nov. 2015.

[38] A. Cecilia, M. Serra, and R. Costa-Castelló, "Nonlinear adaptive observation of the liquid water saturation in polymer electrolyte membrane fuel cells," J. Power Sources, vol. 492, p. 229641, 2021.

[39] A. Astolfi, D. Karagiannis, and R. Ortega, Nonlinear and adaptive control with applications. Springer Science \& Business Media, 2007.

[40] J. Gauthier and G. Bornard, "Observability for anyu(t)of a class of nonlinear systems," IEEE Trans. Autom. Control, vol. 26, no. 4, pp. 922-926, Aug. 1981.
[41] L. B. Freidovich and H. K. Khalil, "Performance recovery of feedbacklinearization-based designs," IEEE Trans. Autom. Control, vol. 53, no. 10, pp. 2324-2334, Nov. 2008.

[42] Z. Jiang, A. Teel, and L. Praly, "Small-gain theorem for iss systems and applications," Math. Control. Signal Sys., vol. 7, pp. 95-120, Jun. 1994.

[43] G.-R. Duan and H.-H. Yu, LMIs in control systems: analysis, design and applications. CRC press, 2013.

[44] D. Astolfi, "Observers and robust output regulation for nonlinear systems," 2016.

[45] R. Olfati-Saber and R. Murray, "Consensus problems in networks of agents with switching topology and time-delays," IEEE Transactions on Automatic Control, vol. 49, no. 9, pp. 1520-1533, 2004.

[46] S.-I. Niculescu, Delay effects on stability: a robust control approach. Springer Science \& Business Media, 2001, vol. 269. 